\title{
Investigating the effect of flow compensation and quantitative susceptibility mapping method on the accuracy of venous susceptibility measurement ${ }^{\sqrt{2}}$
}

\author{
Ronja C. Berg a,*, Christine Preibisch ${ }^{\mathrm{a}, \mathrm{b}, \mathrm{c}}$, David L. Thomas ${ }^{\mathrm{d}, \mathrm{e}, \mathrm{f}}$, Karin Shmueli ${ }^{\mathrm{g}}$, \\ Emma Biondetti ${ }^{\mathrm{g}, \mathrm{h}, \mathrm{l}}$ \\ ${ }^{a}$ Technical University of Munich, School of Medicine, Klinikum rechts der Isar, Department of Diagnostic and Interventional Neuroradiology, Munich, Germany \\ ${ }^{\mathrm{b}}$ Technical University of Munich, School of Medicine, Klinikum rechts der Isar, TUM Neuroimaging Center, Ismaninger Str. 22, 81675 Munich, Germany \\ ${ }^{\mathrm{c}}$ Technical University of Munich, School of Medicine, Klinikum rechts der Isar, Clinic for Neurology, Ismaninger Str. 22, 81675 Munich, Munich, Germany \\ ${ }^{\mathrm{d}}$ Department of Brain Repair and Rehabilitation, UCL Queen Square Institute of Neurology, University College London, London WC1N 3AR, United Kingdom \\ ${ }^{\mathrm{e}}$ Dementia Research Centre, UCL Queen Square Institute of Neurology, University College London, London WC1N 3AR, United Kingdom \\ ${ }^{\mathrm{f}}$ Wellcome Centre for Human Neuroimaging, UCL Queen Square Institute of Neurology, University College London, London WC1N $3 A R$, United Kingdom \\ ${ }^{g}$ Department of Medical Physics and Biomedical Engineering, University College London, London WC1E 6BT, United Kingdom \\ ${ }^{\mathrm{h}}$ Institut du Cerveau - ICM, Centre de NeuroImagerie de Recherche - CENIR, Team "Movement Investigations and Therapeutics" (MOV'IT), INSERM U 1127, CNRS \\ UMR 7225, Sorbonne Université, Paris, France
}

\section{A R T I C L E I N F O}

\section{Keywords:}

Quantitative susceptibility mapping

Venous oxygenation

Flow compensation

Vein segmentation

Sequence design

Image processing

\begin{abstract}
A B S T R A C T
Quantitative susceptibility mapping (QSM) is a promising non-invasive method for obtaining information relating to oxygen metabolism. However, the optimal acquisition sequence and QSM reconstruction method for reliable venous susceptibility measurements are unknown. Full flow compensation is generally recommended to correct for the influence of venous blood flow, although the effect of flow compensation on the accuracy of venous susceptibility values has not been systematically evaluated. In this study, we investigated the effect of different acquisition sequences, including different flow compensation schemes, and different QSM reconstruction methods on venous susceptibilities.
\end{abstract}

Ten healthy subjects were scanned with five or six distinct QSM sequence designs using monopolar readout gradients and different flow compensation schemes. All data sets were processed using six different QSM pipelines and venous blood susceptibility was evaluated in whole-brain segmentations of the venous vasculature and single veins. The quality of vein segmentations and the accuracy of venous susceptibility values were analyzed and compared between all combinations of sequences and reconstruction methods.

Abbreviations: $\chi$, magnetic susceptibility; ANOVA, analysis of variance; CS, Compressed SENSE; dTIK, direct Tikhonov; GRE, gradient-recalled echo; ICVs, internal

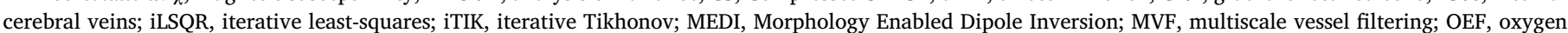

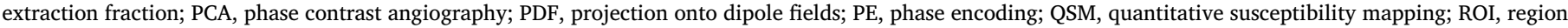

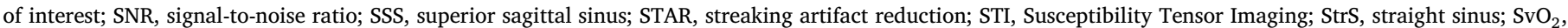

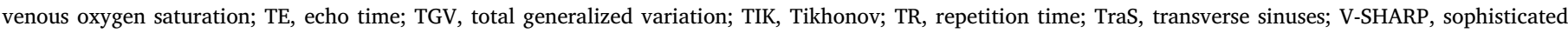
harmonic artifact reduction for phase data with variable kernel sizes.

Funding information: Ronja Berg is supported by a Ph.D. grant from the Friedrich-Ebert-Stiftung. Dr. Christine Preibisch received a grant from the German

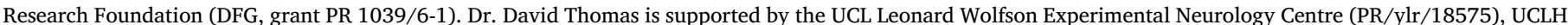

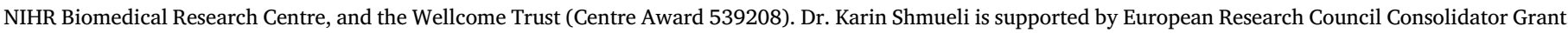

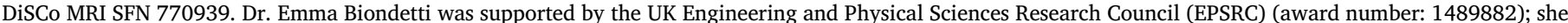

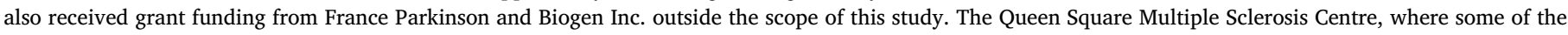

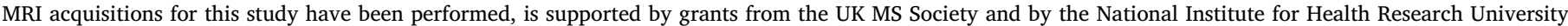
College London Hospitals Biomedical Research Centre.

* Corresponding author.

E-mail addresses: ronja.berg@tum.de (R.C. Berg), preibisch@tum.de (C. Preibisch), d.thomas@ucl.ac.uk (D.L. Thomas), k.shmueli@ucl.ac.uk (K. Shmueli), emma.biondetti@unich.it (E. Biondetti).

1 Present address: Institute for Advanced Biomedical Technologies, Department of Neuroscience, Imaging and Clinical Sciences, “D’Annunzio University" of ChietiPescara, 66100 Chieti, Italy 
The influence of the QSM reconstruction method on average venous susceptibility values was found to be 2.711.6 times greater than the influence of the acquisition sequence, including flow compensation. The majority of the investigated QSM reconstruction methods tended to underestimate venous susceptibility values in the vein segmentations that were obtained.

In summary, we found that multi-echo gradient-echo acquisition sequences without full flow compensation yielded venous susceptibility values comparable to sequences with full flow compensation. However, the QSM reconstruction method had a great influence on susceptibility values and thus needs to be selected carefully for accurate venous QSM.

\section{Introduction}

Venous quantitative susceptibility mapping (QSM) aims to quantify the magnetic susceptibility $(\chi)$ of venous blood based on the phase of the gradient-recalled echo (GRE) magnetic resonance imaging (MRI) signal, which reflects the paramagnetic susceptibility of deoxygenated hemoglobin and venous oxygen saturation $\left(\mathrm{SvO}_{2}\right)$

(Jain et al., 2012; Spees et al., 2001; Weisskoff and Kiihne, 1992). $\mathrm{SvO}_{2}$ is linearly related to $\chi$ of blood and can be calculated as (Fan et al., 2020; Fan et al., 2016; McFadden et al., 2021; Weisskoff and Kiihne, 1992):

$S v O_{2}=1-\left(\Delta \chi_{\text {blood }}-\Delta \chi_{\text {oxy }} * H c t\right) /\left(\Delta \chi_{d o} * H c t\right)$

where $\Delta \chi_{\text {blood }}$ is the measured susceptibility difference between blood and water (or soft tissue), $\Delta \chi_{o x y}$ the constant susceptibility shift of fully oxygenated blood relative to water, $\Delta \chi_{d o}$ the known susceptibility difference between fully oxygenated and fully deoxygenated red blood cells, and $\mathrm{Hct}$ the hematocrit. Venous QSM is suitable for measuring $\mathrm{SvO}_{2}$ in regions containing purely venous voxels. Depending on the chosen image resolution, this assumption is most often met in large cerebral veins.

Based on Eq. (1), previous studies have successfully quantified $\mathrm{SvO}_{2}$ in healthy subjects (Fan et al., 2014; Ward et al., 2017; Xu et al., 2014) and patients with cerebrovascular disease (Biondetti et al., 2019; Fan et al., 2020; Schneider et al., 2020). For assessing brain oxygenation, QSM represents a non-invasive and more feasible alternative to the gold standard ${ }^{15}$ O PET (Mintun et al., 1984). Indeed, PET employs short-lived ${ }^{15} \mathrm{O}$ radiotracers that require on-site production by a cyclotron, whereas QSM exploits the oxygenation status of hemoglobin, a naturally occurring endogenous contrast mechanism (Cho et al., 2020; Fan et al., 2015; Kudo et al., 2016). However, for venous QSM, neither the acquisition sequence nor the image processing pipeline has yet been standardized (Bilgic et al., 2020; Langkammer et al., 2018).

Spins moving during signal encoding gradients accumulate additional non-zero phase, which results in flow-induced signal loss. Flow compensation involves applying gradient lobes before readout to compensate for this additional motion-induced dephasing and restore zero phase at the echo time for spins moving at constant velocity or acceleration. Therefore, it has been suggested that GRE images acquired for venous QSM require full first-order (i.e., velocity) flow compensation (Brown et al., 2014). Indeed, in the absence of flow compensation, vessels containing flowing blood can appear displaced on GRE magnitude (Deistung et al., 2009), GRE phase (Xu et al., 2014), SWI (Deistung et al., 2009), and QSM images (Biondetti et al., 2020), potentially affecting the accuracy of both image-based vessel segmentation and venous susceptibility estimation inside segmented vessels. Fully flow-compensated sequences aim to suppress the additional phase induced by flowing spins (velocity range in intracranial veins: $10-25 \mathrm{~cm} / \mathrm{s}$ (Stolz et al., 1999)) at each echo time (TE) and along all three signal encoding directions of the Cartesian k-space trajectory.

Acquiring multi-echo 3D GRE images for QSM is desirable because it enables optimization of the signal-to-noise ratio (SNR) in multiple tissue types simultaneously (Haacke et al., 2015; Wu et al., 2012a) and fitting over echo times for accurate field map estimation for QSM
(Biondetti et al., 2020). However, commercially available scanner options typically enable full flow compensation only for single-echo protocols or at the first TE of multi-echo protocols. Moreover, the longer TE images of a multi-echo GRE protocol are usually only flow-compensated along the frequency-encoding direction (Denk and Rauscher, 2010; Gilbert et al., 2012). Therefore, pulse sequence programming is required to enable acquisition of fully flow-compensated multi-echo GRE images (Deistung et al., 2009; Xu et al., 2014), limiting the use of these sequences in clinical settings.

In addition to image acquisition, the QSM reconstruction method is expected to impact on measurement accuracy for venous susceptibility. While optimization and standardization of QSM reconstruction algorithms and processing pipelines is an active area of research within the QSM community (Bilgic et al., 2020; Langkammer et al., 2018; Robinson et al., 2017; Schweser et al., 2017), only a few studies have proposed methods specifically optimized for venous QSM reconstruction (Biondetti et al., 2019; Haacke et al., 2015; Liu et al., 2013; Wei et al., 2015; Xu et al., 2014). Critically, the effect of distinct QSM reconstruction pipelines on the accuracy of venous QSM has never been systematically compared.

In this study, we systematically assessed the effect of flow compensation as well as different QSM reconstruction methods on the accuracy of measured venous susceptibility values in both automated whole-brain vein segmentations and single large vein segmentations. To this end, we set up distinct 3D multi-echo GRE sequences with flow compensation applied at all TEs, at the first TE only, or without any flow compensation. Furthermore, because time constraints are known to limit the clinical applicability of multi-echo QSM sequences, we investigated the possibility of using a state-of-the-art acceleration technique, namely Compressed SENSE (Geerts-Ossevoort et al., 2018), compared to the standard SENSE technique (Pruessmann et al., 1999). To independently assess venous anatomy and facilitate vein segmentation, we also acquired phase contrast angiography (PCA) with velocity encoding targeting venous flow. With respect to QSM reconstruction pipelines, we focused primarily on methods featured in the most popular toolboxes available for QSM, since these can be employed by non-specialist users and are therefore feasible in clinical settings. To evaluate their performance, these non-optimized pipelines were compared to a QSM processing method that had been specifically optimized for venous QSM in a previous study (Biondetti et al., 2019) and to an iterative version of the same method (Karsa et al., 2020).

\section{Methods}

\subsection{Subjects}

This study was approved by the local medical ethical committee at the Klinikum rechts der Isar, Technical University of Munich (TUM). After providing informed written consent for participation in this study, ten healthy volunteers (five females, age range: $22-50$ years, average age: 29 years) underwent MRI at the Department of Neuroradiology, Klinikum rechts der Isar, TUM. 


\section{Table 1}

Key acquisition parameters and flow compensation schemes for all of the 3D GRE sequences for QSM and the 3D GRE phase contrast angiography (PCA). Parameters deviating from the most common value are marked in bold. Differences between sequences relevant for QSM include differences in the flow compensation scheme (influencing the bandwidth / water-fat shift), the number of echoes and echo times, and the image acceleration technique (influencing the scan duration). Common parameters were $1 \times 1 \times 1 \mathrm{~mm}^{3}$ resolution, $246 \times 188 \times 144 \mathrm{~mm}^{3}$ field-of-view, transverse slice orientation, and right-left fold-over direction. TE: echo time, $\Delta$ TE: echo spacing, TR: repetition time, PC: phase contrast, RL: right-left, FH: foot-head, Full-FC: Full multi-echo FC, TE1-FC: conventional FC for the first echo only, TE1-FC-CS: first-echo FC with CS acceleration, No-FC: no FC with echoes matched to the Full-FC sequence, No-FC-7ech: no FC with maximum number (7) of TEs, No-FC-3ech: no FC with 3 echoes maximizing SNR.

\begin{tabular}{|c|c|c|c|c|c|c|c|}
\hline & Full-FC & TE1-FC & TE1-FC-CS & No-FC & No-FC-7ech & No-FC-3ech & PCA \\
\hline Acceleration & \multicolumn{7}{|c|}{ SENSE, RL: 2, FH: 1 SENSE, RL: 2, FH: 1 Co } \\
\hline \# echoes & 4 & 4 & 4 & 4 & 7 & 3 & 1 \\
\hline $\mathrm{TE} 1 / \Delta \mathrm{TE}[\mathrm{ms}]$ & $5.7 / 11$ & $5.7 / 11$ & $5.7 / 11$ & $5.7 / 11$ & shortest $(3.4 / 6.1)$ & $7 / 14$ & 6.7 \\
\hline TR $[\mathrm{ms}]$ & shortest (43) & shortest (43) & shortest (43) & shortest (44) & shortest (44) & shortest (43) & shortest (11) \\
\hline \multicolumn{2}{|c|}{ Monopolar gradients (flyback) yes } & yes & yes & yes & yes & yes & - \\
\hline Flip angle $\left[{ }^{\circ}\right]$ & 17 & 17 & 17 & 17 & 17 & 17 & 10 \\
\hline Water-fat shift [pix] & 2.068 & 2.068 & 2.068 & 3.396 & 2.068 & $\max (5.352)$ & $\max (2.585)$ \\
\hline Bandwidth $[\mathrm{Hz}]$ & 210.0 & 210.0 & 210.0 & 127.9 & 210.0 & $\min (81.2)$ & $\min (168)$ \\
\hline Flow compensation (FC) & \multicolumn{2}{|c|}{ Full multi-echo FC First-echo FC } & First-echo FC & No FC & No FC & No FC & First-echo FC \\
\hline Scan duration [min:s] & $9: 40$ & $9: 40$ & $5: 37$ & $10: 01$ & $9: 59$ & $9: 44$ & $10: 12$ \\
\hline $\mathrm{PC} \mathrm{V} \mathrm{V}_{\mathrm{enc}}[\mathrm{cm} / \mathrm{s}]$ & - & - & - & - & - & - & 30 \\
\hline \# data sets & 10 & 10 & 10 & 10 & 10 & 4 & 10 \\
\hline
\end{tabular}

To investigate reproducibility across different MRI systems from the same vendor, data from five healthy volunteers (three females, age range: $30-54$ years, average age: 44 years) who had been scanned previously in a pilot study were included in the analysis. The pilot study was performed at the Queen Square Multiple Sclerosis Centre (University College London (UCL), UCL Institute of Neurology, London, UK). This study was approved by the local ethics review board and all subjects provided written informed consent.

\subsection{Image acquisition}

Data acquisition was performed on a Philips Ingenia Elition X $3 \mathrm{~T}$ MR system (Philips Healthcare, R5.6.1.0, Best, NL) using a 32-channel head coil. All imaging sequences for QSM and PCA were based on a 3D GRE sequence. For QSM, these sequences contained several echoes to optimize SNR in different tissues. Images were acquired in transverse orientation with $1 \mathrm{~mm}$ isotropic resolution (compatible with the diameter of large veins (Curé et al., 1994; Durst et al., 2016)), $246 \times 188 \times 144 \mathrm{~mm}^{3}$ fieldof-view (read $x$ phase encoding $(\mathrm{PE})_{1} \times \mathrm{PE}_{2}$ ), with frequency encoding in the anterior-posterior direction and a right-left primary phase encoding (fold-over) direction. SENSE (2-fold acceleration in the $\mathrm{PE}_{1}$ direction) was used as the standard imaging acceleration technique. All GRE sequences for QSM had monopolar (flyback) readout gradients enabled, flip angle $17^{\circ}$, and the shortest achievable repetition time (which was 43 or $44 \mathrm{~ms}$ for all sequences). Other acquisition parameters, namely the number of echoes and the readout bandwidth, were optimized in line with each sequence's flow compensation scheme (see Table 1 for a detailed overview). Additionally, in one sequence, Compressed SENSE (CS) (Geerts-Ossevoort et al., 2018) was applied (4-fold acceleration) to evaluate the effect of reducing acquisition time by sparse sampling of $\mathrm{k}$ space. The following flow compensation schemes were tested: full flow compensation for all echoes and along all encoding directions (Full-FC) as in a previous study (Xu et al., 2014); a conventional flow compensation scheme for the first echo only as implemented by the vendor using SENSE (TE1-FC) or Compressed SENSE (TE1-FC-CS); and no flow compensation with TEs matched either to the Full-FC sequence (No-FC) or with the maximum number of TEs within the repetition time (TR) (NoFC-7ech). Finally, to investigate the effect of maximizing the SNR over the same TR on multi-echo QSM accuracy, a sequence was implemented with no flow compensation and three TEs (No-FC-3ech), which is the minimum number of TEs for multi-echo QSM using the Morphology Enabled Dipole Inversion (MEDI) toolbox (see Section 2.3.2). All sequences were run on all subjects except No-FC-3ech, which, because of time restrictions, was only tested on four subjects. Additionally, PCA data were acquired to independently locate and segment major cerebral veins. The
PC velocity encoding threshold $\left(V_{\text {enc }}\right)$ was chosen in line with the expected peak systolic forward velocity of flow in the major intracranial veins and sinuses, which, on average, ranges between 7 and $20 \mathrm{~cm} / \mathrm{s}$ in healthy subjects (Stolz et al., 1999). The GRE sequence implementation with full flow compensation was enabled by research software made available by the scanner manufacturer.

The data of the pilot study had been acquired on a Philips Achieva $3 \mathrm{~T}$ system (software version R3.2.1) using a 32-channel head coil. The acquisition protocol comprised three of the sequences used in the main study: Full-FC, No-FC, and No-FC-7ech with identical parameter settings except for a $1.1 \times 1.1 \times 1.1 \mathrm{~mm}^{3}$ resolution and a $246 \times 180 \times 144 \mathrm{~mm}^{3}$ field-of-view.

\subsection{Image processing and analysis}

Where not stated otherwise, all data processing and analyses were performed using MATLAB (R2020a, The Mathworks, Natick, MA, United States).

\subsubsection{Brain mask calculation}

For each subject, brain masks for QSM were calculated based on the longest-TE magnitude image from each sequence. This enabled exclusion of anatomical areas near air-tissue interfaces, which cause artifacts due to signal dropout at longer TEs. The magnitude images were segmented into tissue compartments using the "segment" module of the Statistical Parametric Mapping toolbox (SPM12; https://www.fil.ion.ucl.ac.uk/spm/software/spm12/) for MATLAB. Each brain mask was calculated as the sum of the gray matter, white matter, and cerebrospinal fluid tissue compartments. In each transverse slice, residual holes in the brain mask were filled using an inhouse script written in MATLAB to evaluate each voxel in the mask in relation to its four direct within-slice neighbors. The superior sagittal sinus was added to the brain mask using ITK-SNAP's semi-automated segmentation tool (Yushkevich et al., 2006) (www.itksnap.org) on the firstecho magnitude image as detailed in a previous study (Biondetti et al., 2019).

\subsubsection{QSM processing pipelines}

Each 3D QSM data set was processed using six different QSM reconstruction pipelines (see Fig. 1 for a schematic overview). Three methods were based on two publicly available toolboxes: the MEDI toolbox (version 11/2017; http://pre.weill.cornell.edu/mri/pages/qsm.html) and the Susceptibility Tensor Imaging (STI) Suite (v3.0_05/2017; https://people.eecs.berkeley.edu/ chunlei.liu/software.html), both with default settings applied. Additionally, we tested total generalized 


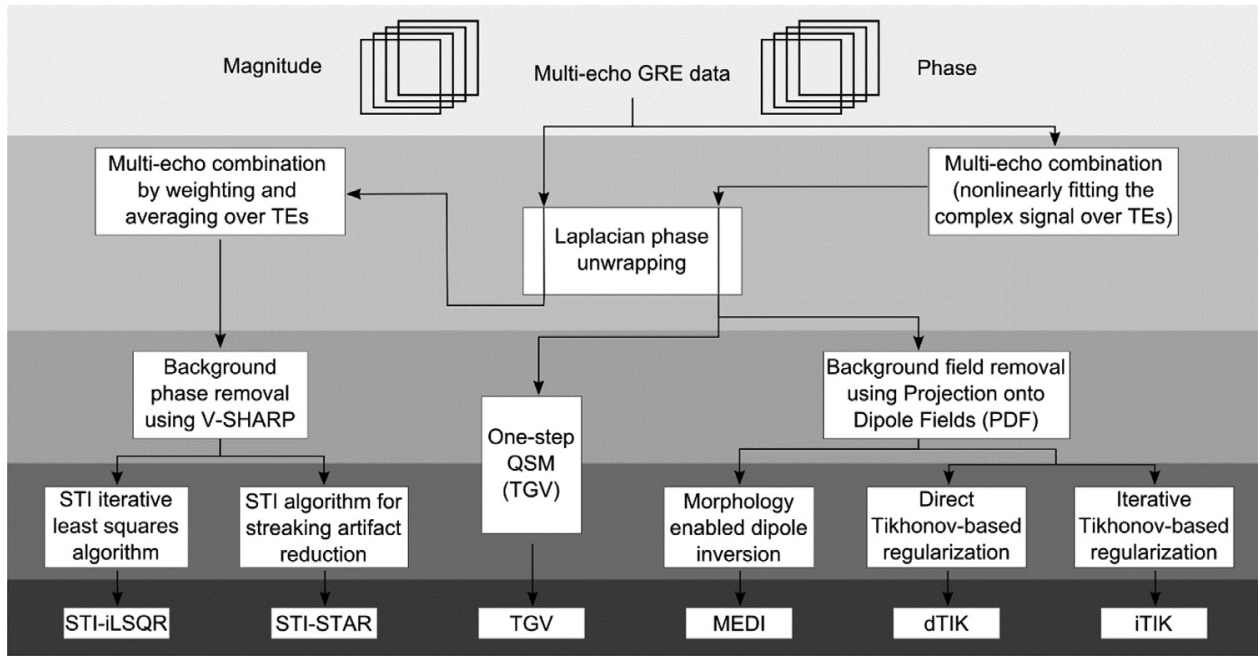

Fig. 1. Schematic diagram of the processing steps in each QSM reconstruction pipeline. The input data consisted of magnitude and phase images from each of the different GRE sequences listed in Table 1. Multi-echo combination was performed after Laplacian phase unwrapping when using STI Suite and before phase unwrapping in all other pipelines. In TGV, background field removal and field-tosusceptibility inversion were performed within the same step. All other QSM pipelines performed these two steps separately. variation (TGV) (Langkammer et al., 2015), a one-step algorithm, and two Tikhonov (TIK)-based QSM reconstruction methods, the latter having been optimized and applied previously for venous QSM (Biondetti et al., 2020; Biondetti et al., 2019). All methods except TGV used the same brain mask, calculated as described above. Calculated susceptibility values were not referenced to a specific brain tissue to avoid combining the effects of acquisition or QSM reconstruction method in a reference region of interest (ROI) with those in venous ROIs.

For multi-step processing using the MEDI toolbox, the total field map was calculated by fitting the complex GRE signal over TEs. Phase unwrapping was performed using a Laplacian-based technique (Schofield and Zhu, 2003) and background fields were removed via projection onto dipole fields (PDF) (de Rochefort et al., 2010; Liu et al., 2011). Local field-to-susceptibility inversion was performed using the Morphology Enabled Dipole Inversion "MEDI_L1" (Liu et al., 2012; Liu et al., 2011) method with a reduced radius of 3 for the spherical mean value operator "SMV" to avoid excessive erosion of the brain mask, and otherwise default values. This included a default value of 1000 for the regularization parameter (recommended by the developers for brain applications and verified by checking several values on these data), since the toolbox did not provide any options for optimizing this parameter

In the STI Suite, Laplacian-based phase unwrapping was applied and the multi-echo unwrapped phase images were then combined via TEweighted averaging (Li et al., 2015; Wei et al., 2015). Background fields were removed using sophisticated harmonic artifact reduction for phase data with variable kernel sizes (V-SHARP) (Li et al., 2011; Wu et al., $2012 b)$. Local field-to-susceptibility inversion was performed using two distinct methods: iterative least-squares (iLSQR) (Li et al., 2011) and an algorithm specifically designed for reducing streaking artifacts potentially arising from high-susceptibility sources such as large veins (streaking artifact reduction, STAR) (Wei et al., 2015).

One-step TGV-based QSM (Langkammer et al., 2015) was performed using Singularity (Sylabs Inc., Albany, CA, USA; https://github.com/ CAIsr/qsm) and the default parameter values $\alpha_{1}, \alpha_{0}=0.0015,0.0005$ based on the criterion that $\alpha_{1}: \alpha_{0}=3: 1$ is optimal for medical imaging applications (Knoll et al., 2011). The inputs for TGV QSM were the field map combined using the nonlinear fitting function in the MEDI toolbox (Liu et al., 2013) and the echo spacing.

The input for the Tikhonov-based QSM calculations was the local field map calculated by the MEDI-based pipeline. Local fieldto-susceptibility inversion was performed using direct Tikhonov regularization (dTIK; https://xip.uclb.com/i/software/mri_qsm_tkd.html) as well as an iterative implementation (iterative Tikhonov, iTIK; https://xip.uclb.com/i/software/mri_qsm_tkd.html), both with correction for susceptibility underestimation. For dTIK, the average optimal regularization parameter "alpha" $\left(\alpha_{d T}\right)$ across subjects was calculated separately for each acquisition sequence using the L-curve method (Hansen and O'Leary, 1993). The values for $\alpha_{d T}$ ranged between 0.070 and 0.074 and can be found in Table S1 in the supplementary material. For iTIK, an empirical fixed value of $\alpha_{i T}=0.05$, recommended by the developer for brain applications, was used as the regularization parameter.

In summary, the processing pipelines differed in three main steps: multi-echo combination, background field removal, and local field-tosusceptibility inversion. A detailed comparison of the individual processing steps within the investigated QSM processing pipelines is shown in Table S2 in the supplementary material.

As some of the QSM reconstruction methods resulted in slightly eroded brain volumes, we calculated a minimum-size brain mask to have a common volume for all our analyses. This minimum-size brain mask was calculated for each acquisition sequence and subject separately as the intersection of brain volumes in each of the processed QSM images.

\subsubsection{Whole-brain vessel filtering}

The automated multiscale vessel filtering (MVF) method (Bazin et al., 2015) (version 3.0.7) from the JIST-LayoutTool (v1.8, 08/2013; https://www.nitrc.org/projects/jist/) of MIPAV (v8.0.2, 02/2018; https://mipav.cit.nih.gov/) was used for whole-volume vessel segmentation of the susceptibility maps from each QSM pipeline. The segmentation was performed using default parameter settings including a recursive ridge filter and a scale number (kernel size) equal to 4 (Biondetti et al., 2019).

\subsubsection{Single-vein segmentation}

Based on the PCA venogram, semi-automated segmentation of major representative veins (superior sagittal sinus (SSS), straight sinus (StrS), transverse sinuses (TraS), and internal cerebral veins (ICVs)) was performed using ITK-SNAP's "active contour segmentation mode" (Bettoni et al., 2018; Law and Chung, 2013). Here, the TraS segment comprised the transverse sinuses, extending into the sigmoid sinuses and the internal jugular veins. This segment was defined to investigate the global performance of venous QSM in the inferior part of the brain. The PCA-based venous ROIs were aligned with each susceptibility map using SPM12 (nearest neighbor interpolation) via rigid alignment of the PCA magnitude image with the corresponding first-echo GRE magnitude image. To mitigate partial volume effects between veins and surrounding brain tissue, the co-registered ROIs of larger veins (i.e., SSS, StrS, and TraS) were eroded with a spherical shaped structural element by one voxel for further analyses. 


\subsubsection{Quantitative analysis}

All vein segmentations (whole-brain and single-veins) were multiplied with the minimum-size brain mask. The average and standard deviation of susceptibility values were calculated in both the masked whole-brain MVF-based and single-vein segmentations. For each susceptibility map, venous density was calculated as the fraction of MVFsegmented venous voxels over the total number of voxels in the brain mask.

To assess the accuracy of MVF-based automated segmentation, two different analyses were performed. First, a consensus segmentation map was calculated from the six MVF-based automated segmentations from different QSM reconstructions per sequence and subject. This consensus map was thresholded at a probability of $50 \%$ resulting in a map of pixels classified as blood vessel in at least three out of the six MVF-based segmentations. The fraction of correctly segmented venous voxels ("true positives") was then determined within this consensus segmentation. Second, the non-eroded PCA-based single-vein ROIs were considered as a gold-standard reference and the fraction of venous voxels correctly detected by the MVF segmentation within these ROIs was calculated.

To assess the similarity between segmentation outcomes across susceptibility maps from different acquisition sequences and reconstruction methods, Sørensen-Dice similarity coefficients were calculated between automated whole-brain segmentations from different susceptibility maps. The coefficients were computed as (Dice, 1945) $\operatorname{DSC}(A, B)=$ $\frac{2 *(A \cap B)}{|\mathrm{A}|+|\mathrm{B}|}$, where $|\mathrm{A}|$ is the number of voxels in the segmentation of susceptibility map (A), $|\mathrm{B}|$ the number of voxels in the segmentation of susceptibility map (B), and $(A \cap B)$ the number of voxels in the intersection of both segmentations. Additionally, Sørensen-Dice similarity coefficients were calculated between each of the automated whole-brain segmentations from different susceptibility maps and the consensus segmentation.

For accurate vein imaging, susceptibility maps need to represent veins as tubular connected structures. To investigate this aspect, the connectedness of voxels within the intersection of the PCA-based segmentation of ICVs and the MVF-segmentation was evaluated. To this end, the "bwconncomp" MATLAB function was utilized to find the largest connected component within the intersection map. The number of voxels within this largest connected component was then divided by the total number of voxels in the PCA-based ICVs segmentations. Additionally, subject-mean values of vessel diameters and partial volume contributions of veins obtained from the MVF algorithm were calculated within the PCA-based single-vein segmentations. Note that "partial volume" $=0$ in a given voxel means absence of vessels in that voxel.

To estimate the respective influence of QSM reconstruction methods and imaging sequences, venous susceptibilities from data acquired with different sequences but reconstructed with the same QSM reconstruction method were averaged to obtain a method-mean venous susceptibility value, $\bar{\chi}_{\text {method }}$. Equivalently, susceptibilities calculated from data acquired with the same sequence but reconstructed with different QSM reconstruction methods were averaged to obtain a sequence-mean venous susceptibility value, $\bar{\chi}_{\text {sequence }}$. The maximal difference between the six method-mean venous susceptibility values $\left(\Delta \bar{\chi}_{\text {method }}\right)$ and the maximal difference between the six sequence-mean venous susceptibility values $\left(\Delta \bar{\chi}_{\text {sequence }}\right)$ were determined, and their ratio was calculated to assess the relative importance of the two factors.

Finally, venous oxygenation values were calculated according to Eq. (1) with $H c t=0.4, \Delta \chi_{d o}=0.27 * 4 \pi \mathrm{ppm}$ (Spees et al., 2001), and $\Delta \chi_{\text {oxy }}=-0.03 * 4 \pi$ ppm (Weisskoff and Kiihne, 1992).

\subsubsection{Statistical analysis}

Statistical analyses were performed using SPSS (IBM Corp. Released 2019. IBM SPSS Statistics for Windows, Version 26.0. Armonk, NY). For whole-brain MVF-based and semi-automated single-vein segmentations, separate two-way repeated measures analyses of variance (ANOVAs) were applied to test whether there was a statistically significant difference in average venous susceptibility when using different imag- ing sequences and applying distinct QSM reconstruction pipelines. The Shapiro-Wilk test and Mauchly's Test of Sphericity were applied to test and verify the normality of each data set's distribution and sphericity, respectively, as requirements for performing the ANOVA. Effect sizes $\left(\eta^{2}\right)$ were calculated as (Lakens, 2013) $\eta^{2}=\frac{S S_{\text {effect }}}{S S_{\text {total }}}$, where $S S_{\text {effect }}$ is the sum of squares of an effect (in this study, e.g., QSM reconstruction method or QSM sequence) and $S S_{\text {total }}$ the total sum of squares (in the study, variability of venous susceptibility values). Simple main effects were analyzed for all average venous susceptibilities of segmentations that showed a significant interaction between both effects (QSM reconstruction method and QSM sequence). Therefore, paired samples $t$-tests were run for all possible combinations of the six QSM reconstruction methods ( 15 per sequence) for the five sequences that were acquired in all ten subjects (75 combinations) and for all pairs of sequences per reconstruction method (60 combinations). The Benjamini-Hochberg procedure was then applied to control for the false discovery rate.

\subsection{Data availability statement}

In line with local ethics guidelines and subject privacy policies, the full set of acquired data are available via a request to the authors, as institutional policies require a formal data sharing agreement. However, an example data set of the participant shown in Figs. 2 to 4 (including defaced raw magnitude and phase images, brain mask, susceptibility maps, multiscale vessel filtering segmentations, and PCA-based singlevein segmentations aligned to the QSM images) can be downloaded via the following link: https://doi.org/10.5281/zenodo.4947237. The full MATLAB code applied for susceptibility map calculation is available upon request and all the QSM reconstruction methods (Fig. 1) are publicly available via the links in Section 2.3.2. Sharing of any sequence modification applied here is limited by a nondisclosure agreement with the scanner manufacturer.

\section{Results}

\subsection{Overall vessel appearance on QSM}

Multi-echo GRE images acquired using the different acquisition sequences appeared similar on visual inspection (Fig. 2). Likewise, differences between reconstructed susceptibility maps from data acquired using different flow compensation schemes appeared small on simple visual inspection (Fig. 3). Conversely, susceptibility maps reconstructed with the six different QSM reconstruction methods exhibited clear differences in overall tissue contrast, extent of brain erosion, also affecting SSS visibility, and vein delineation performance (Fig. 3). Some streaking artifacts were found in the MEDI reconstructions (Fig. 3, MEDI, sagittal slices), while the Tikhonov-based reconstructions seemed to contain residual low-frequency (background) field contributions (Fig. 3, dTIK and iTIK). Generally, in the MEDI, TGV, and dTIK susceptibility maps, veins had a higher contrast relative to the surrounding brain tissue, but substantially decreased susceptibility values in the immediate vicinity of venous areas (Fig. 3, arrowheads). In the STI-STAR susceptibility maps, veins often appeared less well defined. Additionally, in some of the susceptibility maps reconstructed with both STI-based methods, the susceptibility in specific veins or venous segments, e.g., within the SSS or StrS, appeared lower than in the surrounding brain tissue (Fig. 3, arrows), resulting in an overall reduced conspicuity of these veins.

\subsection{Performance of automated whole-brain vessel segmentation}

For the same acquisition sequence, the number and location of automatically segmented venous voxels differed across QSM reconstructions (Fig. 4A). While about 10-25\% of automatically MVF-based segmented venous voxels were recognized by the MVF algorithm in all six susceptibility maps from the same sequence (Fig. 4A, blue squares and yellow arrows), others were only segmented in some or just a single QSM 

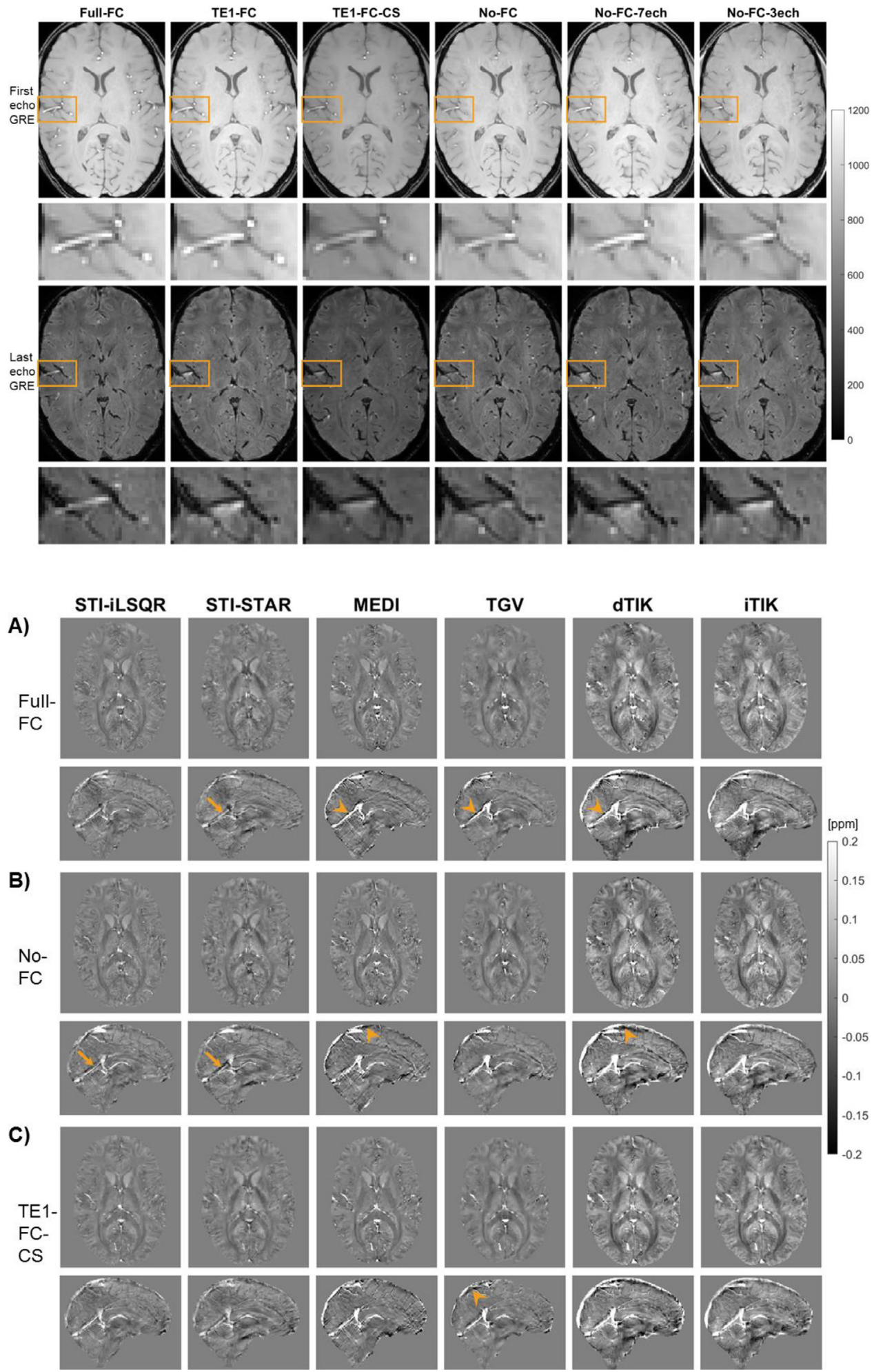

Fig. 2. Representative GRE magnitude images of the first (top rows) and last (bottom rows) echo for each of the six sequences. Similar slices (not perfectly aligned) were selected for each sequence. Zoomed-in regions of the first echo and last echo GRE (orange rectangles) are shown in the second and fourth row, respectively. The magnitude image quality was comparable across all multi-echo GRE imaging sequences. All images are scaled in arbitrary units. The magnitude image brightness can be affected by automatic adjustment of the scanner.

Fig. 3. Representative transverse and sagittal slices of the same healthy subject shown in Fig. 2 are shown for all susceptibility maps reconstructed with the six different QSM pipelines (columns) for three of the imaging sequences. Similar transverse and sagittal slices were selected for (A) Full-FC, (B) No-FC, and (C) TE1-FC-CS sequences. Differences in the susceptibility maps can be seen between the six reconstruction methods including the degree of SSS erosion, substantially decreased susceptibility values around veins (arrowheads), and reduced susceptibility values within venous areas (arrows). reconstruction (Fig. 4A, arrowheads). MEDI and TGV had the highest fractions $(\sim 80 \%)$ of correctly segmented voxels compared to the consensus segmentations, while STI-STAR had the lowest fractions ( $\sim 45 \%)$ of correctly detected voxels (Fig. 5A). Susceptibility maps reconstructed with both TIK-based reconstructions showed high fractions of true positive voxels $(\sim 70 \%)$ in the segmentation (Fig. $5 \mathrm{~A})$ and resulted in the highest Dice similarity scores (0.7-0.9) with the consensus segmentation (Fig. 5B).
Dice scores quantifying the relative overlap between individual whole-brain segmentations are reported in Supplementary Fig. S3. For all imaging sequences, the highest Dice scores (range: 0.5-0.7) were found between the two TIK-based susceptibility maps, whereas the lowest Dice scores (range: 0.2-0.45) were found between STI-STAR and all non-STI-based pipelines (Supplementary Fig. S3).

Automated whole-brain segmentations were compared to independent PCA-based semi-automated segmentations of single representative 

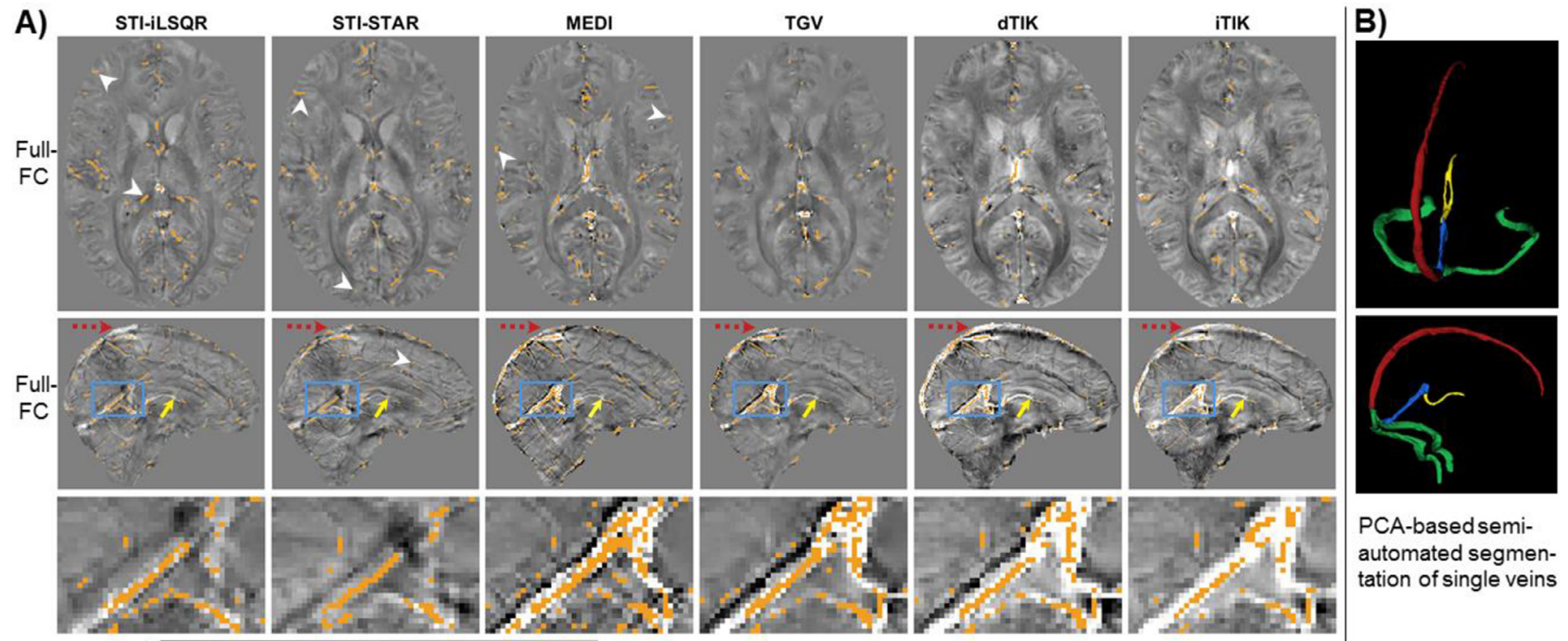

PCA-based semiautomated segmentation of single veins

noxels segmented with MVF in each individual susceptibility map

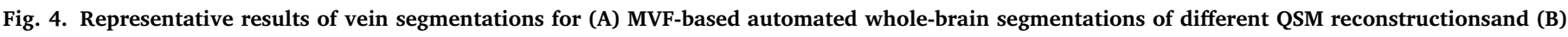

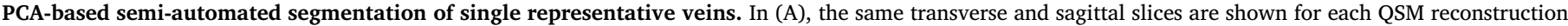

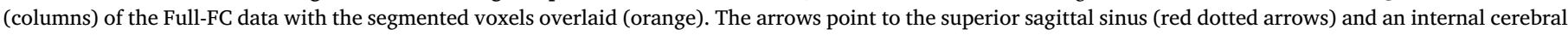

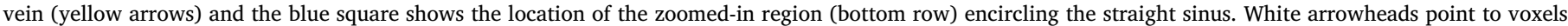

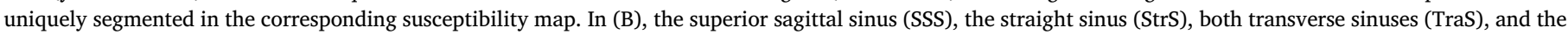
internal cerebral veins (ICVs) are colored in red, blue, green, and yellow, respectively.

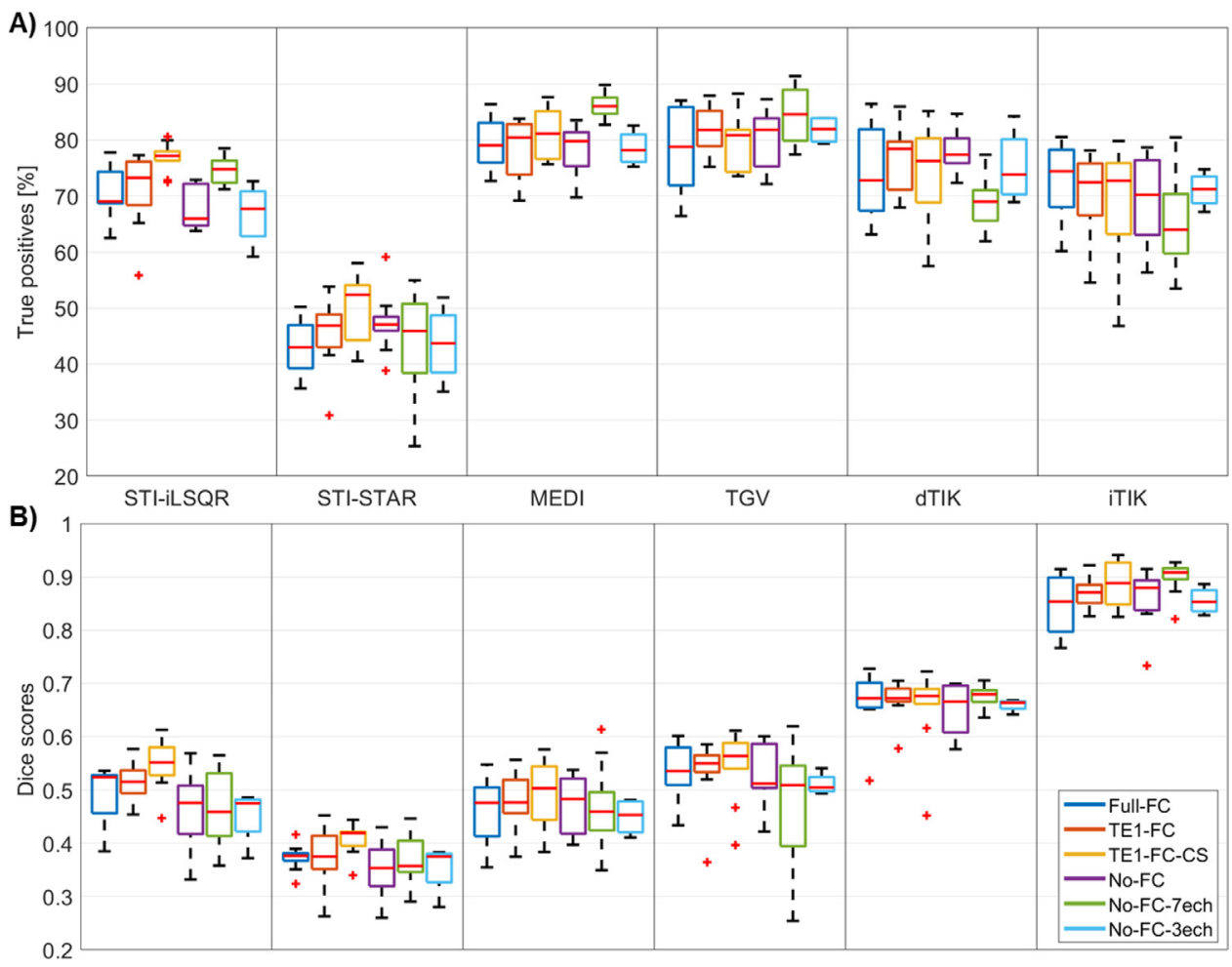

Fig. 5. Agreement of automated wholebrain segmentations with a consensus segmentation map across sequences and processing pipelines. For each QSM reconstruction method and acquisition sequence, panel (A) shows boxplots of the fraction of true positives (correctly segmented voxels) of the various segmentations, i.e., voxels conforming with the consensus segmentation. In panel (B), Sørensen-Dice similarity coefficients between whole-brain segmentations of susceptibility maps reconstructed with different QSM reconstruction methods and the consensus segmentation are shown. Each acquisition sequence is represented using a different color and the six QSM reconstruction methods are grouped by column. In both panels, the boxplots represent distributions across subjects.

veins (Fig. 4B). MEDI and TGV reconstructions had the highest fractions of voxels correctly segmented within the representative veins (Fig. 6). Subject-mean vessel diameters of the PCA-based single-vein ROIs ranged between $2 \mathrm{~mm}$ for the ICVs and $3 \mathrm{~mm}$ for the TraS (Supplementary Fig. S4, A-D). Additionally, the partial volume contributions of veins were determined within the single-vein ROIs. The contribution of veins in these segmentations ranged mainly between 70-85\% (Supplementary Fig. S4, E-H). Slightly lower partial volume contributions of veins and thus higher contributions of surrounding tissue were found for STI-STAR $(60-70 \%)$ and in superficial veins for TGV $(\sim 60 \%$ in the SSS).

Supplementary Fig. S5 shows the fraction of connected voxels in the MVF-based segmentations within the corresponding semi-automated ICVs segmentation. STI-STAR reconstructions had the lowest fractions of connected voxels (average: 7-9\%), STI-iLSQR and both Tikhonovbased reconstructions had a slightly higher fraction (average: 9-28\%), 

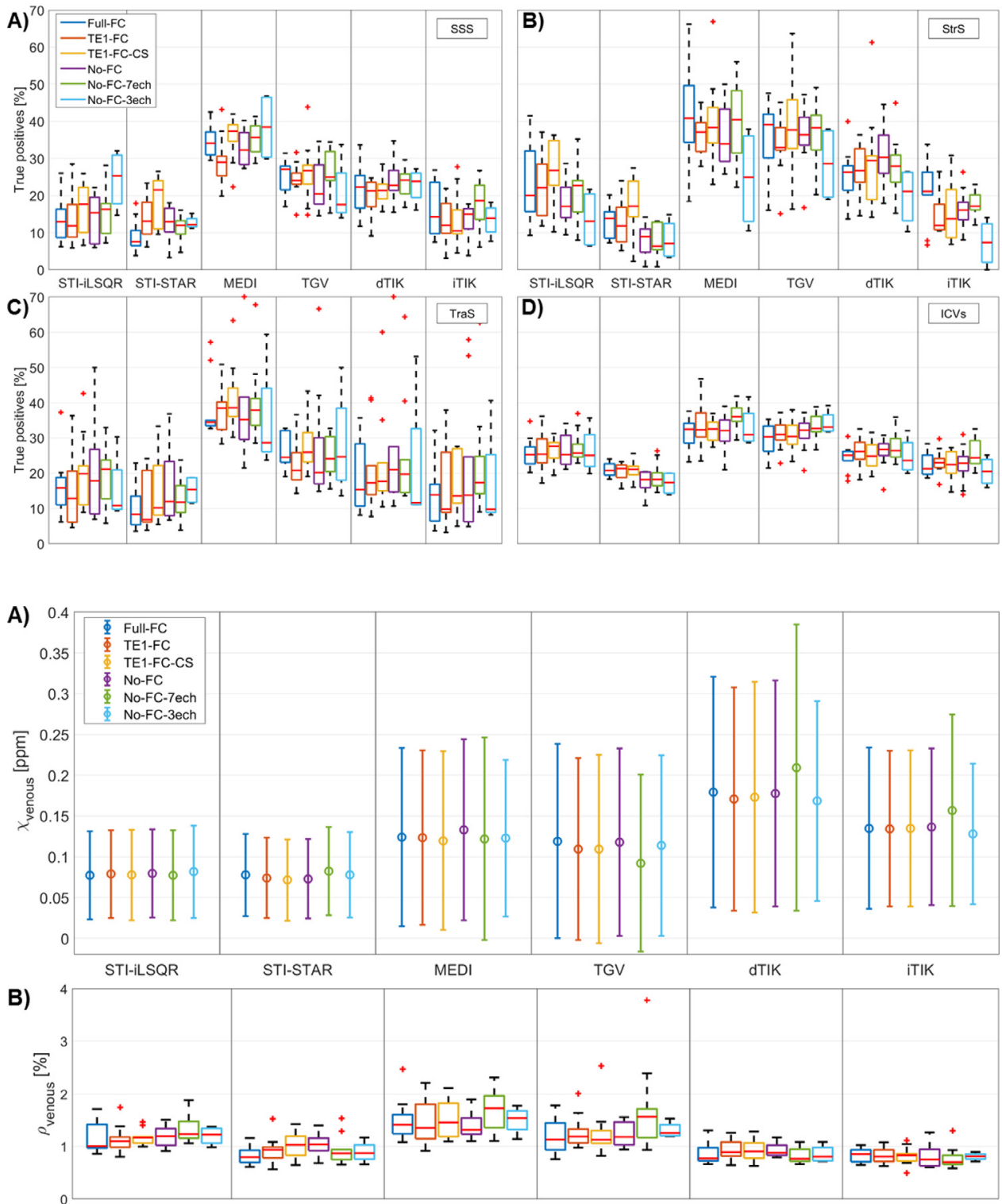

Fig. 6. True positives (correctly segmented voxels) of the automated whole-brain segmentations identified in the gold-standard PCA-based segmentations of single representative veins within (A) the superior sagittal sinus (SSS), (B) the straight sinus (StrS), (C) both transverse sinuses (TraS), and (D) the internal cerebral veins (ICVs). The four different (bilateral) vein segmentations were obtained using semi-automated segmentation on PCA images and used as a gold standard after co-registration to each individual susceptibility map. Each acquisition sequence is represented using a different color and the six QSM reconstruction methods are grouped by column. In all four panels, the boxplots represent distributions across subjects.
Fig. 7. Whole-brain (A) mean and standard deviations of venous susceptibility values across subjects and (B) subject-mean venous densities for the six acquisition sequences (different colors) and six QSM reconstruction methods (columns). Venous susceptibility and venous density values were calculated across all voxels obtained from multiscale vessel filtering on individual susceptibility maps within a common minimum-size brain mask. Differences in mean venous susceptibility and subject-mean venous density are greater for different QSM reconstruction methods than for different acquisition settings. whereas MEDI and TGV reconstructions had the highest fraction of connected voxels (average: $22-35 \%$ ).

Similar results were obtained from automated whole-brain vessel segmentation of the pilot study data (including five different subjects imaged on another scanner model from the same vendor). The highest fractions of correctly segmented venous voxels within the consensus segmentation map (Supplementary Fig. S6A) were again highest for MEDI and TGV and lowest for STI-STAR reconstructions, while the Dice scores were highest for iTIK susceptibility maps (Supplementary Fig. S6B). Furthermore, Dice scores between pairs of QSM reconstruction methods in the pilot study data (Supplementary Fig. S7) correlated well with the corresponding Dice scores of the main study (Supplementary Fig. S3). Analyses requiring PCA for segmentation could not be performed on the pilot study data, since PCA images were only acquired in three of the five subjects, resulting in a sample size too small for meaningful statistical analysis.

\subsection{Susceptibility quantification inside venous segmentations}

In all subjects, STI-based reconstructions yielded the lowest average susceptibility values for both the whole-brain automated vein segmentations (Fig. 7A) and the single-vein semi-automated segmentations (Fig. 8). Notably, TIK-based versus STI-based reconstructions yielded similar whole-brain venous densities $(\sim 0.7-1.0 \%)$, but consistently higher average susceptibility values (Fig. 7). MEDI and TGV reconstructions resulted in slightly higher venous densities in the brain ( $1.0-1.4 \%)$ (Fig. 7B). In all venous segmentations, dTIK reconstructions resulted in the highest venous susceptibility values and thereby in the lowest $\mathrm{SvO}_{2}$ values (Supplementary Table S8), but also had the most variable venous susceptibility values (Figs. 7A and 8).

Similar average susceptibility values and venous densities $(\sim 0.6-$ $1.3 \%$ ) were obtained from five different subjects acquired on another scanner model from the same vendor (Supplementary Fig. S9).

Two-way repeated measures ANOVA with Greenhouse-Geisser correction for non-sphericity revealed that significant differences in the average whole-brain venous susceptibility were linked to both the type of imaging sequence $(F(4,36)=4.19, p=0.007$, $\left.\eta^{2}=0.006\right)$ and the QSM reconstruction method employed $(F(2.36$, $\left.20.32)=222.10, p<0.001, \eta^{2}=0.866\right)$. Additionally, there was a statistically significant interaction between the effects of imaging sequence and QSM reconstruction method on venous susceptibility $(F(5.38$, 48.38) $=5.13, p=0.001, \eta^{2}=0.028$ ). Similar results were found for 

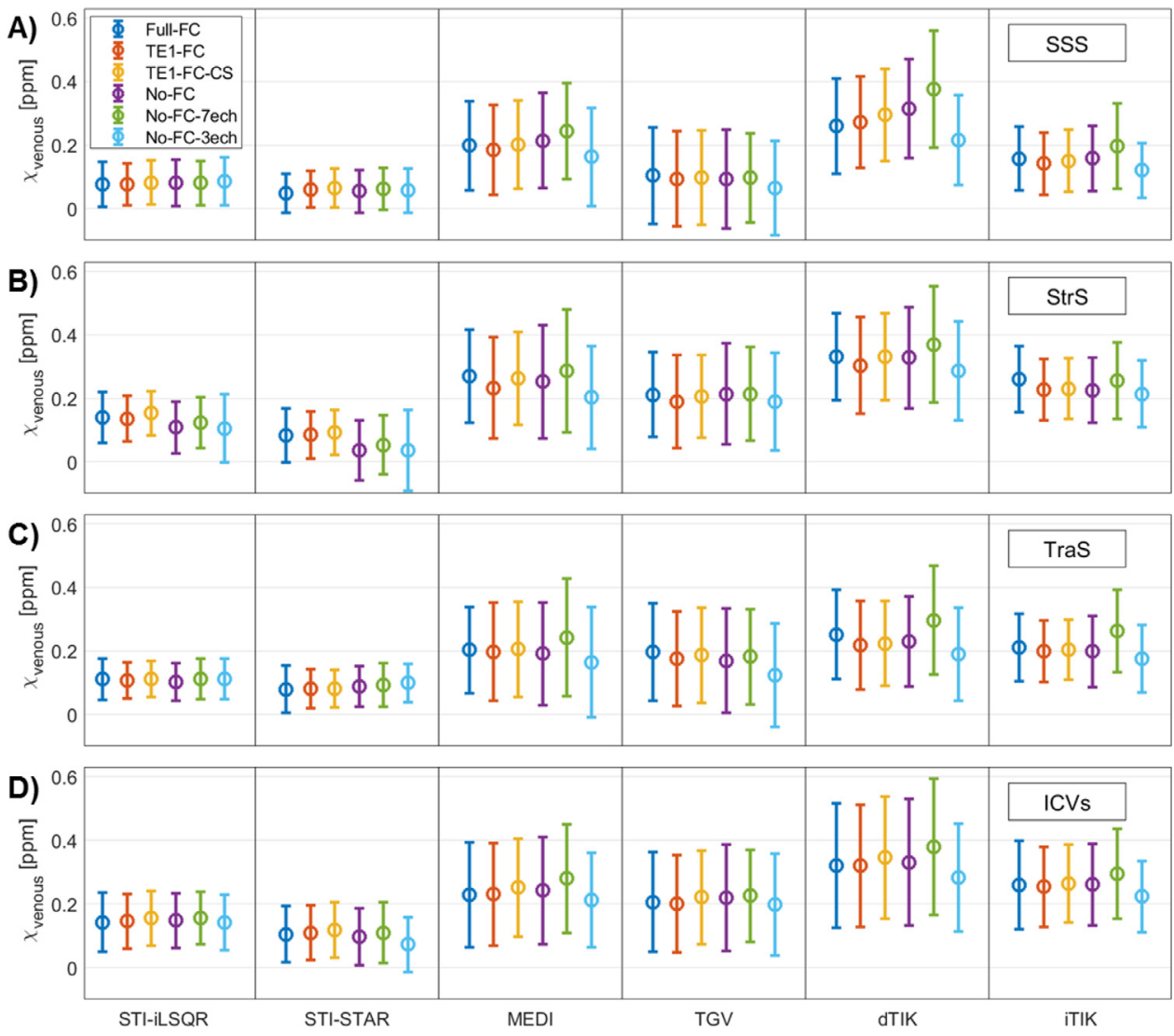

Fig. 8. Mean and standard deviations of venous susceptibility values in single-vein segmentations of (A) the superior sagittal sinus (SSS), (B) the straight sinus (StrS), (C) both transverse sinuses (TraS), and (D) the internal cerebral veins (ICVs). Data are shown for the six acquisition sequences (different colors) and six QSM reconstruction methods (columns). Mean venous susceptibility was calculated across all voxels obtained from semiautomated segmentation on PCA images after co-registration to each individual susceptibility map. Differences in mean venous susceptibility are greater for different QSM reconstruction methods than for different acquisition settings.

Table 2

Maximal differences between the method-mean $\bar{\chi}_{\text {method }}$ and sequence-mean $\bar{\chi}_{\text {sequence }}$ venous susceptibility values and their quotient. The method-mean values were calculated by averaging over venous susceptibility values acquired with six different sequences but reconstructed with the same QSM reconstruction method. Accordingly, sequence-mean values were calculated by averaging over venous susceptibility values acquired with the same sequence but reconstructed with six different QSM reconstruction methods.

\begin{tabular}{llllll}
\hline Maximal average differences in $\bar{\chi}$-venous & MVF & SSS & StrS & TraS & ICVs \\
\hline $\boldsymbol{\Delta} \bar{\chi}_{\text {sequence }}[\mathrm{ppm}]$ & 0.009 & 0.058 & 0.045 & 0.054 & 0.053 \\
$\boldsymbol{\Delta} \bar{\chi}_{\text {method }}[\mathrm{ppm}]$ & 0.104 & 0.236 & 0.263 & 0.143 & 0.231 \\
$\boldsymbol{\Delta} \bar{\chi}_{\text {method }} / \boldsymbol{\Delta} \bar{\chi}_{\text {sequence }}$ & 11.56 & 4.07 & 5.84 & 2.65 & 4.36 \\
\hline
\end{tabular}

the average susceptibility in single-vein segmentations (Supplementary Table S10).

Paired samples $t$-tests with Benjamini-Hochberg correction for a false discovery rate revealed that most average venous susceptibility values calculated with different QSM reconstruction methods were significantly different from each other (Supplementary Table S11). However, for various acquisition sequences and across several venous segments, no significant differences in average venous susceptibility were found between iTIK and MEDI as well as between iTIK and TGV. Furthermore, some pairs of reconstruction methods did not show statistically significantly different mean susceptibility values within single venous segments, such as STI-iLSQR and TGV within the SSS or MEDI and dTIK within the TraS segmentations.

Much smaller differences in venous susceptibility values were found between data acquired with different imaging sequences than across the six QSM reconstruction methods (Figs. 7A and 8). However, venous susceptibility values acquired with the No-FC-7ech sequence were frequently slightly increased compared to other sequences. Similarly, paired samples $t$-tests with Benjamini-Hochberg correction comparing mean susceptibilities from different acquisition sequences revealed only a few statistically significant differences (Supplementary Table S12). These differences were mainly found between the No-FC-7ech sequence and any of the other sequences.

For all single-vein segmentations, $\Delta \bar{\chi}_{\text {sequence }}$, the maximal difference between the six sequence-mean venous susceptibility values $\left(\bar{\chi}_{\text {sequence }}\right)$, was almost constant (varying between 0.05 and $0.06 \mathrm{ppm}$ ), while $\Delta \bar{\chi}_{\text {method }}$, the maximal difference between the six method-mean venous susceptibility values $\left(\bar{\chi}_{\text {method }}\right)$, varied more strongly across the segmentations (between 0.14 and $0.26 \mathrm{ppm}$ ) (Table 2). These results show that the influence of the QSM reconstruction method on the average venous susceptibility values was much stronger than the influence of the acquisition sequence, ranging from a factor of 2.7 (TraS) to 5.8 (StrS). Wholebrain segmentations, which contained many more voxels compared to single-vein segmentations, resulted in smaller maximal differences between sequence-mean and method-mean susceptibilities. However, in the whole-brain segmentations, the influence of the QSM reconstruction method on the average venous susceptibility was 11.6 times larger than the influence of the sequence (Table 2), which concurs with a factor of 12.4 obtained for the pilot study data acquired on a different scanner model (Supplementary Table S13). 


\section{Discussion}

This study aimed to compare acquisition sequences and QSM reconstruction pipelines for venous QSM. The effect of flow compensation during image acquisition was evaluated by applying sequences incorporating different flow compensation schemes versus no flow compensation when using monopolar readout gradients. The effect of using different QSM reconstruction methods was evaluated by testing pipelines from popular QSM toolboxes compared to pipelines previously optimized for venous QSM. The effects of acquisition sequence and QSM reconstruction method on the accuracy of venous susceptibility values were assessed by applying all combinations of sequences and QSM pipelines to the multi-echo GRE data acquired in ten healthy volunteers. The results were validated in a second smaller cohort of five healthy volunteers who were scanned using a subset of the same acquisition sequences at a different site.

\subsection{Influence of the imaging sequence on venous QSM}

Generally, there was a high similarity in the visual appearance of susceptibility maps, venous susceptibility values, and the performance of whole-brain segmentation between acquisition sequences. Slight differences were only found for the two sequences without flow compensation (No-FC-3ech and No-FC-7ech) that had a different number of echoes from all other sequences (which had four echoes). Susceptibility maps reconstructed from the No-FC-3ech sequence (maximizing SNR over the minimum number of TEs needed for multi-echo fitting using MEDI) resulted in slightly lower average venous susceptibility values within single-vein segmentations (Fig. 8) and in slightly lower variances in susceptibility in the whole-brain segmentations (for the MEDI, dTIK, and iTIK pipelines) (Fig. 7A). However, the smaller sample size acquired with this sequence (four subjects) did not allow a direct comparison with other sequences (ten subjects).

Sequences with four echoes had more consistent mean susceptibility values and it is possible that including more echoes in the nonlinear fit (used for all but the STI-based methods) slightly improved the accuracy of venous QSM. The No-FC-7ech sequence resulted in slightly higher venous susceptibility values compared to all other sequences (Figs. 7A and 8). Here, a possible cause for measuring higher venous susceptibility values may be phase accumulation due to the lack of flow compensation. Moreover, compared to the two other sequences without any type of flow compensation, the No-FC-7ech sequence had a lower signal-tonoise ratio (due to its higher bandwidth). Venous susceptibility values differed least between the fully flow compensated sequence (Full-FC) and the sequences with only first-echo flow compensation (TE1-FC and TE1-FC-CS) or the sequence without flow compensation and matched echo times (No-FC). This suggests that the number of echoes had more of an effect on the susceptibility values than the flow compensation scheme.

Generally, different flow compensation implementations yielded comparable venous susceptibility values. Even in the largest cerebral veins, such as the superior sagittal sinus or the transverse sinuses, venous susceptibility values were not statistically significantly different between sequences with and without flow compensation. It is possible that the monopolar readout gradients employed in all imaging sequences mitigated the lack of flow compensation by effectively applying a partial form of incidental flow compensation in the frequency encoding direction, even when full flow compensation was not explicitly implemented. However, the effect of monopolar read gradients on flow compensation in the three encoding directions could be further evaluated, e.g., using a flow phantom experiment similar to Xu et al. (2014). For this purpose, three water-filled tubes, each connected to a pump generating constant flow with known velocities, could be oriented parallel to the three encoding directions. Nonetheless, sequences without flow compensation or with only first-echo flow compensation appear just as suitable for quantitative analyses as sequences with full flow compensation when monopolar read gradients are used.

No significant differences in image quality and venous susceptibility were detected when comparing the Compressed SENSE-accelerated sequence (TE1-FC-CS) with the other SENSE-accelerated sequences. Furthermore, the TE1-FC-CS sequence enabled reliable whole-brain segmentations, resulting in slightly higher Dice similarity scores with the consensus segmentation compared to other sequences for most of the QSM reconstruction methods (Fig. 5B). Therefore, our results suggest that, for venous QSM, Compressed SENSE could be used to obtain clinically feasible scan times (5-6 min) without introducing significant venous susceptibility differences compared to SENSE-accelerated sequences.

\subsection{Influence of the QSM reconstruction pipeline on venous QSM}

Our results show that the choice of QSM processing pipeline is crucial for correctly reconstructing high susceptibility values in veins. The QSM reconstruction method had a much (2.7 to 11.6 times) greater effect on the average venous susceptibility than the acquisition sequence in general or the use of flow compensation in particular (Table 2, Figs. 7A and 8). These findings were reproduced using data acquired on a different scanner model from the same vendor (Supplementary Table S13 and Supplementary Fig. S9). Furthermore, different vein segmentation approaches did not influence our results: similar trends were seen when using either automated whole-brain or semi-automated single-vein segmentation methods (Figs. 7A and 8).

\subsection{Accuracy of automated whole-brain segmentations}

Higher fractions of correctly segmented voxels within the consensus segmentation (Fig. 5A) and within the gold-standard single-vein segmentations (Fig. 6), and higher fractions of connected voxels within the PCA-segmented ICVs (Supplementary Fig. S5) were regarded as indicators of higher quality of the automated whole-brain vein segmentation. Higher Dice overlaps with the whole-brain consensus segmentation (Fig. 5B) and lower contributions of partial volume effects in the PCA-based single vein segmentations (higher influence of vein, Supplementary Fig. S4, E-H) were regarded as additional indicators of more accurate vein segmentation. The latter is the case, as partial volume effects of surrounding tissue can artificially decrease the susceptibilities in veins. Based on these indicators, QSM reconstruction methods enabling more accurate automatic whole-brain segmentation (TIK-based methods and MEDI) also resulted in the highest venous susceptibilities within both the whole-brain and single-vein segmentations (Figs. 7A and 8). These same methods also resulted in fewer partial volume effects between veins and the surrounding brain tissue (Supplementary Fig. S4, E-H). In contrast, susceptibility maps from STI-STAR, which showed the lowest average venous susceptibility values, appeared to yield inaccurate automated whole-brain segmentations. STI-STAR's lower venous susceptibility values and the smoother tissue-vein boundaries probably impeded detectability by the MVF algorithm and led to increased partial volume contributions from surrounding tissue.

However, it must be noted that partial volume estimates (and, thus, diameter estimates) were probably inaccurate in large superficial veins, as all QSM processing methods eroded the brain volume. Such brain erosion was found to be most prominent in susceptibility maps reconstructed with TGV, resulting in fewer true positives and greater partial volume contributions from surrounding tissue in the SSS and the TraS segmentations (Supplementary Fig. S4, E-H). TGV-based susceptibility maps were slightly more affected by brain mask erosion during processing compared to susceptibility maps reconstructed with other methods and this is likely to have impeded accurate representation and reliable automated detection of superficial veins. 


\subsection{Differences between processing pipelines}

Susceptibility maps reconstructed using methods from the STI Suite were found to differ most from the other QSM reconstructions, e.g., resulting in comparatively low venous susceptibility values (Figs. 7A and 8) and low fractions of correctly segmented voxels (Fig. 6). These differences between susceptibility maps calculated using STI-based pipelines and those reconstructed using all other pipelines could be caused by differences in multi-echo GRE signal combination. For the STI-based pipelines, the phase at each echo was first unwrapped and then averaged over all echoes using a TE-based weighting approach (Li et al., 2015; Wei et al., 2015). Conversely, all other pipelines combined the complex GRE signal by nonlinear fitting over TEs before performing spatial phase unwrapping (Liu et al., 2013). Unlike fitting over TEs, averaging over TEs does not allow removal of phase offsets from the fitted field map. Moreover, Laplacian-based phase unwrapping could alter the linearity of the signal phase over time by inherently performing some degree of background field suppression, thus yielding inaccurate values if the phase is combined over TEs after Laplacian unwrapping (Biondetti et al., 2016; Schweser et al., 2013). These two aspects could have jointly contributed to the generally lower accuracy of STI-based venous QSM. Additionally, in the STI-STAR pipeline, high-susceptibility areas are reconstructed separately from the surrounding tissue by applying a two-step algorithm (Wei et al., 2015). In the first step, the regularization parameter is tuned to "high-susceptibility sources", which are reconstructed separately, and then the forward model (Marques and Bowtell, 2005) is applied to estimate and remove field variations from these sources from the original field map. In the second step, "low-susceptibility sources" are reconstructed in the rest of the brain by tuning the regularization parameter to brain parenchyma (Wei et al., 2015). However, the first regularization parameter was originally tuned for detecting hemorrhages, which are characterized by much higher susceptibility values (up to $\sim 1 \mathrm{ppm}$ ) than normal appearing veins $(\sim 0.3-0.4 \mathrm{ppm})$ (Wei et al., 2015). Thus, in our study, it is possible that voxels within a normal venous susceptibility range were largely omitted in the first step of the STI-STAR method, but then over-regularized in the second step, resulting in unrealistically low venous susceptibilities. Note that tuning the regularization parameters to different values was not possible because the corresponding function in the STI toolbox is designated as private.

Generally, differences between QSM reconstruction methods in the regularization used in the local field-to-susceptibility inversion step can influence the appearance of susceptibility maps and the variability of susceptibility values. A lower degree of regularization could explain the higher variance of venous susceptibility found for dTIK and the lower overall homogeneity generally seen in TIK-based susceptibility maps possibly caused by residual background fields. Additionally, differences in the background field removal method affect the field maps and could thus influence the resulting susceptibility maps, especially in regions near the brain mask boundary such as superficial veins (Schweser et al., 2017). A previous study found that V-SHARP (used in both STI-based QSM reconstructions) resulted in less accurate field maps near the brain surface (Schweser et al., 2017) compared to other methods such as PDF (used in MEDI and TIK-based QSM reconstructions). This could have been another factor contributing to the lower accuracy of STI-based venous QSM.

\subsection{Quantification of venous oxygenation}

In this study, unreferenced susceptibility values were used to calculate $\mathrm{SvO}_{2}$ values, as referencing would have introduced an additional component of variation between the methods. Evaluation of susceptibility values in an established reference region, i.e., the lateral ventricles within the CSF (Straub et al., 2017), revealed slight differences between QSM processing methods. However, referencing the susceptibility maps to the average susceptibility within the lateral ventricles did not affect the overall trends and findings of our study. As CSF susceptibility val- ues within the reference region were small for all reconstruction techniques, and referencing did not influence our overall results, we chose to omit this step in our comparison study. However, in quantitative studies comparing venous oxygenation values in different subjects or subject cohorts using a fixed QSM processing method, susceptibility values could be referenced before calculating $\mathrm{SvO}_{2}$ values, although this may slightly increase the variance across subjects.

In most of the combinations of acquisition sequence and QSM reconstruction method, the venous susceptibility tended to be underestimated and consequently the resulting $\mathrm{SvO}_{2}$ was overestimated compared to literature values (Biondetti et al., 2019; Fan et al., 2014; Xu et al., 2014). Whole-brain $\mathrm{SvO}_{2}$ was overestimated even using MEDI and TGV, which seemed promising for automatically quantifying whole-brain venous oxygenation since they provided the most accurate automatic segmentation results. QSM reconstructions from dTIK yielded venous oxygenation values (Supplementary Table S8) closest to venous oxygenation obtained by gold-standard PET measurements of the oxygen extraction fraction ( $\mathrm{OEF}, \mathrm{SvO}_{2}=1-\mathrm{OEF}$ ). In all single-vein segmentations, the average venous susceptibility in dTIK QSM ranged between $0.19 \mathrm{ppm}$ and 0.38 ppm corresponding to an $\mathrm{SvO}_{2}$ of 60.9-74.9\%, which is in good agreement with literature values measured using ${ }^{15} \mathrm{O}$ PET (i.e., 59-72\%) (Cho et al., 2020; Ibaraki et al., 2008; Ishii et al., 1996) and MRI based on (apparent) transverse relaxation times $\left(\mathrm{T}_{2}{ }^{*}\right.$ and $\mathrm{T}_{2}$ ) (i.e., 63-70.6\%) (Barhoum et al., 2015; Cho et al., 2020; Fan et al., 2016; Jain et al., 2013). In MEDI and iTIK reconstructions, accurate $\mathrm{SvO}_{2}$ values were only found in the StrS and ICVs segmentations, whereas $\mathrm{SvO}_{2}$ values in the SSS and TraS were overestimated.

Generally, whole-brain venous susceptibility distributions within dTIK reconstructions had the highest variance (Fig. 7A), suggesting less efficient noise or streaking artifact reduction compared to other pipelines. Nevertheless, the good correspondence of $\mathrm{SvO}_{2}$ from dTIK (and partly also from iTIK or MEDI) QSM with $\mathrm{SvO}_{2}$ measured using ${ }^{15} \mathrm{O}$ PET suggests that susceptibility maps with the best overall visual appearance might not provide the most accurate susceptibility quantification (and $\mathrm{SvO}_{2}$ measurement) in veins. Similarly, the QSM reconstruction challenge 2.0 showed that errors in the estimation of susceptibility values in the veins were particularly high, even for many of the topranked QSM algorithms (Bilgic et al., 2020). These findings indicate that the quantification of susceptibilities in venous blood remains prone to errors and needs further optimization.

\subsection{Limitations and recommendations for future studies}

To investigate differences in susceptibility values within the same venous voxels, all evaluations were performed using a minimum-size brain mask, calculated as the intersection of brain volumes in each of the processed QSM images. Even though the same original brain mask was used as an input for all QSM processing methods, the output brain regions differed slightly between the QSM pipelines. These differences arose as some methods (especially TGV and MEDI) inherently applied additional steps that include erosion, which are not easily modifiable, especially for non-expert users. However, after applying the minimum-size brain mask to each individual susceptibility map, the semi-automated SSS and TraS segmentations were significantly reduced in size and a large portion of these superficial vessels was excluded from both the whole-brain and the single-vein analyses. When designing the image reconstruction pipeline, future studies for venous QSM should account for the degree of brain erosion. For venous QSM, further work is needed to evaluate the accuracy of methods that do not require brain erosion during background field removal (Liu et al., 2017) and could thus enable segmentation of the entire venous vasculature.

Partial volume effects of vessels with the surrounding brain tissue are a common confounding factor in venous QSM leading to decreased venous susceptibility. This problem was addressed automatically for MVF-based whole-brain segmentations through a model-based approach (Bazin et al., 2015) and using erosion for single-vein segmentations. 
However, in smaller veins, erosion was not feasible since it would have removed most segmented venous voxels. Thus, our single-vein analyses were mainly limited to large dural sinuses and one ROI drawn on smaller veins (the ICVs) that was analyzed without applying erosion.

Flow acceleration effects on the measured venous susceptibility and $\mathrm{SvO}_{2}$ values were not investigated. However, it has been shown using the related method of susceptibility-based oximetry (Cheng et al., 2021) that flow acceleration could influence $\mathrm{SvO}_{2}$ measurements in a TE-dependent fashion when applying flow compensation along the frequency encoding direction at the first TE, similar to our TE1-FC and TE1-FC-CS sequences. Although the present study highlights a substantially larger effect of image processing than imaging sequence on venous QSM, further investigation of TE-dependent acceleration effects on velocity-compensated venous QSM measurements is required.

In this study, image acquisition was limited to a single vendor and one field strength. Future studies on $3 \mathrm{~T}$ systems from different vendors are needed to evaluate, for example, the feasibility of multi-echo GRE MRI using compressed sensing. Moreover, since $\mathrm{T}_{2}{ }^{*}$ values shorten with increasing field strength and the optimal phase SNR for a given tissue is achieved when TE equals $\mathrm{T}_{2}{ }^{*}$ of that tissue (Wu et al., 2012a), ultra-high fields (e.g., $7 \mathrm{~T}$ ) could be used to further reduce scan time for venous QSM. In fact, venous QSM has already been successfully used to image the brain vasculature at $7 \mathrm{~T}$ (Huck et al., 2019). Future studies could build on these results to investigate the potential reduction in acquisition time achievable by reaching the optimal phase SNR at shorter TEs.

\section{Conclusion}

To conclude, the effect of different QSM reconstruction methods on mean venous susceptibility values was several times greater than the effect of varying acquisition settings including flow compensation. This indicates that specific optimization of QSM algorithms and pipelines is essential for accurate venous QSM and, in turn, to enable reproducible venous susceptibility quantification across studies. Future venous QSM studies need to carefully select their QSM reconstruction method based on their individual research question and to consider that the high susceptibilities in veins could most accurately be quantified by QSM reconstruction methods that do not necessarily provide the "best-looking" maps in other tissue types. Towards this aim, processing pipelines based on Tikhonov regularization or, alternatively, the MEDI toolbox appeared to provide a good overall performance in both successful automated vein segmentation and accurate quantification of venous susceptibility.

\section{Data and code availability statement}

In line with local ethics guidelines and subject privacy policies, the full set of acquired data is available via a request to the authors, as institutional policies require a formal data sharing agreement. However, an example data set of the participant shown in Figs. 2 to 4 (including defaced raw magnitude and phase images, brain mask, susceptibility maps, multiscale vessel filtering segmentations, and PCA-based singlevein segmentations aligned to the QSM images) can be downloaded via the following link: https://doi.org/10.5281/zenodo.4947237. The full MATLAB code applied for susceptibility map calculation is available upon request and all the QSM reconstruction methods are publicly available via the links provided in the Methods section. Sharing of any sequence modification applied here is limited by a nondisclosure agreement with the scanner manufacturer.

\section{Declaration of Competing Interest}

Emma Biondetti received grant funding from Biogen Inc. and France Parkinson unrelated to the scope of this study.

\section{Credit authorship contribution statement}

Ronja C. Berg: Methodology, Software, Validation, Formal analysis, Investigation, Writing - original draft, Visualization. Christine Preibisch: Conceptualization, Writing - review \& editing, Supervision, Funding acquisition. David L. Thomas: Writing - review \& editing, Supervision. Karin Shmueli: Writing - review \& editing, Supervision, Funding acquisition. Emma Biondetti: Conceptualization, Methodology, Investigation, Writing - review \& editing, Supervision.

\section{Acknowledgements}

We thank Dr. Guillaume Gilbert (MR Clinical Science, Philips Healthcare, Markham, Canada) for providing the software for full multi-echo flow compensation and Dr. Anita Karsa (Department of Medical Physics and Biomedical Engineering, University College London (UCL), London, UK) for discussion on implementing the iterative Tikhonov method. We thank Dr. Jakob Meineke (Philips Research, Hamburg, Germany) and Dr. Andreas Hock (Philips Healthcare, Hamburg, Germany) for their support and discussion during preparatory test scans and analyses at the Technical University of Munich. We also thank Dr. Marco Battiston, Dr. Francesco Grussu, and Dr. Marios Yiannakas (NMR Research Unit, Queen Square Multiple Sclerosis Centre, UCL Queen Square Institute of Neurology, UCL, London, UK) and Dr. Rosa Cortese and Dr. Floriana De Angelis (Queen Square MS Centre, UCL Queen Square Institute of Neurology, UCL, London, UK) for their help during the MRI scans at UCL. We are indebted to Prof. Claudia Gandini Wheeler-Kingshott (NMR Research Unit, Queen Square MS Centre, UCL Queen Square Institute of Neurology, UCL, London, UK) for her support with the MRI experiments performed at the Queen Square MS Centre, UCL.

\section{Supplementary materials}

Supplementary material associated with this article can be found, in the online version, at doi:10.1016/j.neuroimage.2021.118399.

\section{References}

Barhoum, S., Rodgers, Z.B., Langham, M., Magland, J.F., Li, C., Wehrli, F.W., 2015. Comparison of MRI methods for measuring whole-brain venous oxygen saturation. Magn. Reson. Med. 73, 2122-2128. doi:10.1002/mrm.25336.

Bazin, P.-L., Fan, A.P., Mianowska, G., Olbrich, A., Schäfer, A., Villringer, A., Gauthier, C.J., 2015. Automated vessel segmentation from quantitative susceptibility maps at 7 Tesla. In: Proceedings of the Twenty-forth Annual Meeting of the ISMRM,(Toronto, ON).

Bettoni, J., Pagé, G., Salsac, A.-V., Constans, J.-M., Testelin, S., Devauchelle, B., Balédent, O., Dakpé, S., 2018. 3T non-injected phase-contrast MRI sequences for the mapping of the external carotid branches: in vivo radio-anatomical pilot study for feasibility analysis. J. Craniomaxillofac. Surg. 46, 98-106. doi:10.1016/j.jcms.2017.09.005.

Bilgic, B., Langkammer, C., Marques, J.P., Meineke, J., Milovic, C., Schweser, F., Committee, Q.C.O., 2020. QSM Reconstruction Challenge 2.0: Design and Report of Results bioRxiv doi:10.1101/2020.11.25.397695.

Biondetti, E., Karsa, A., Thomas, D.L., Shmueli, K., 2020. Investigating the accuracy and precision of TE-dependent versus multi-echo QSM using Laplacian-based methods at 3 T. Magn. Reson. Med. 84, 3040-3053. doi:10.1002/mrm. 28331.

Biondetti, E., Rojas-Villabona, A., Sokolska, M., Pizzini, F.B., Jäger, H.R., Thomas, D.L., Shmueli, K., 2019. Investigating the oxygenation of brain arteriovenous malformations using quantitative susceptibility mapping. Neuroimage 199, 440-453. doi:10.1016/j.neuroimage.2019.05.014.

Biondetti, E., Thomas, D.L., Shmueli, K., 2016. Application of laplacian-based methods to multi-echo phase data for accurate susceptibility mapping. In: Proceedings of the 24th International Society for Magnetic Resonance in Medicine. ISMRM.

Brown, R.W., Cheng, Y.-C.N., Haacke, E.M., Thompson, M.R., Venkatesan, R., 2014. Magnetic Resonance Imaging: Physical Principles and Sequence Design. John Wiley \& Sons.

Cheng, C.M., Chou, C.C., Yeh, T.C., Chung, H.W., 2021. Measurements of venous oxygen saturation in the superior sagittal sinus using conventional 3D multiple gradientecho MRI: effects of flow velocity and acceleration. Magn. Reson. Med. 85, 995-1003. doi:10.1002/mrm.28474.

Cho, J., Lee, J., An, H., Goyal, M.S., Su, Y., Wang, Y., 2020. Cerebral oxygen extraction fraction (OEF): Comparison of challenge-free gradient echo QSM+qBOLD (QQ) with 150 PET in healthy adults. J. Cereb. Blood Flow Metab. doi:10.1177/0271678X20973951, 0271678X20973951.

Curé, J.K., Van Tassel, P., Smith, M.T., 1994. Normal and variant anatomy of the dural venous sinuses. In: Seminars in ultrasound, CT, and MR, pp. 499-519. doi:10.1016/S0887-2171(05)80019-8. 
de Rochefort, L., Liu, T., Kressler, B., Liu, J., Spincemaille, P., Lebon, V., Wu, J., Wang, Y., 2010. Quantitative susceptibility map reconstruction from MR phase data using bayesian regularization: validation and application to brain imaging. Magn. Reson. Med. 63, 194-206. doi:10.1002/mrm.22187.

Deistung, A., Dittrich, E., Sedlacik, J., Rauscher, A., Reichenbach, J.R., 2009. ToF-SWI: simultaneous time of flight and fully flow compensated susceptibility weighted imaging. J. Magn. Reson. Imaging 29, 1478-1484. doi:10.1002/jmri.21673.

Denk, C., Rauscher, A., 2010. Susceptibility weighted imaging with multiple echoes. J. Magn. Reson. Imaging 31, 185-191. doi:10.1002/jmri.21995.

Dice, L.R., 1945. Measures of the amount of ecologic association between species. Ecology 26, 297-302. doi:10.2307/1932409.

Durst, C.R., Ornan, D.A., Reardon, M.A., Mehndiratta, P., Mukherjee, S., Starke, R.M., Wintermark, M., Evans, A., Jensen, M.E., Crowley, R.W., 2016. Prevalence of dural venous sinus stenosis and hypoplasia in a generalized population. J. Neurointerv. Surg. 8, 1173-1177 http://dx.doi.org/10.1136/neurintsurg-2015-012147.

Fan, A.P., Bilgic, B., Gagnon, L., Witzel, T., Bhat, H., Rosen, B.R., Adalsteinsson, E., 2014. Quantitative oxygenation venography from MRI phase. Magn. Reson. Med. 72, 149159. doi:10.1002/mrm.24918.

Fan, A.P., Govindarajan, S.T., Kinkel, R.P., Madigan, N.K., Nielsen, A.S., Benner, T., Tinelli, E., Rosen, B.R., Adalsteinsson, E., Mainero, C., 2015. Quantitative oxygen extraction fraction from 7-Tesla MRI phase: reproducibility and application in multiple sclerosis. J. Cereb. Blood Flow Metab. 35, 131-139. doi:10.1038/jcbfm.2014.187.

Fan, A.P., Khalil, A.A., Fiebach, J.B., Zaharchuk, G., Villringer, A., Villringer, K., Gauthier, C.J., 2020. Elevated brain oxygen extraction fraction measured by MRI susceptibility relates to perfusion status in acute ischemic stroke. J. Cereb. Blood Flow Metab. 40, 539-551. doi:10.1177/0271678X19827944.

Fan, A.P., Schäfer, A., Huber, L., Lampe, L., von Smuda, S., Möller, H.E., Villringer, A., Gauthier, C.J., 2016. Baseline oxygenation in the brain: correlation between respiratory-calibration and susceptibility methods. Neuroimage 125, 920-931. doi:10.1016/j.neuroimage.2015.11.007.

Geerts-Ossevoort, L., De Weerdt, E., Duijndam, A., van IJperen, G., Peeters, H., Doneva, M., Nijenhuis, M., Huang, A., 2018. Compressed SENSE Speed Done Right. Every Time. Koninklijke Philips N.V.

Gilbert, G., Savard, G., Bard, C., Beaudoin, G., 2012. Quantitative comparison between a multiecho sequence and a single-echo sequence for susceptibility-weighted phase imaging. Magn. Reson. Imaging 30, 722-730. doi:10.1016/j.mri.2012.02.008.

Haacke, E.M., Liu, S., Buch, S., Zheng, W., Wu, D., Ye, Y., 2015. Quantitative susceptibility mapping: current status and future directions. Magn. Reson. Imaging 33, 1-25. doi:10.1016/j.mri.2014.09.004.

Hansen, P.C., O'Leary, D.P., 1993. The use of the L-curve in the regularization of discrete ill-posed problems. SIAM J. Sci. Comput. 14, 1487-1503. doi:10.1137/0914086.

Huck, J., Wanner, Y., Fan, A.P., Jäger, A.-T., Grahl, S., Schneider, U., Villringer, A., Steele, C.J., Tardif, C.L., Bazin, P.-L., 2019. High resolution atlas of the venous brain vasculature from $7 \mathrm{~T}$ quantitative susceptibility maps. Brain Struct. Funct. 224, 24672485. doi:10.1007/s00429-019-01919-4.

Ibaraki, M., Miura, S., Shimosegawa, E., Sugawara, S., Mizuta, T., Ishikawa, A., Amano, M., 2008. Quantification of cerebral blood flow and oxygen metabolism with 3-dimensional PET and 15O: validation by comparison with 2-dimensional PET. J. Nucl. Med. 49, 50-59. doi:10.2967/jnumed.107.044008.

Ishii, K., Sasaki, M., Kitagaki, H., Sakamoto, S., Yamaji, S., Maeda, K., 1996. Regional difference in cerebral blood flow and oxidative metabolism in human cortex. J. Nucl. Med. 37, 1086-1088.

Jain, V., Abdulmalik, O., Propert, K.J., Wehrli, F.W., 2012. Investigating the magnetic susceptibility properties of fresh human blood for noninvasive oxygen saturation quantification. Magn. Reson. Med. 68, 863-867. doi:10.1002/mrm.23282.

Jain, V., Magland, J., Langham, M., Wehrli, F.W., 2013. High temporal resolution in vivo blood oximetry via projection-based T2 measurement. Magn. Reson. Med. 70, 785790. doi:10.1002/mrm.24519.

Karsa, A., Punwani, S., Shmueli, K., 2020. An optimized and highly repeatable MRI acquisition and processing pipeline for quantitative susceptibility mapping in the headand-neck region. Magn. Reson. Med. 84, 3206-3222. doi:10.1002/mrm.28377.

Knoll, F., Bredies, K., Pock, T., Stollberger, R., 2011. Second order total generalized variation (TGV) for MRI. Magn. Reson. Med. 65, 480-491. doi:10.1002/mrm.22595.

Kudo, K., Liu, T., Murakami, T., Goodwin, J., Uwano, I., Yamashita, F., Higuchi, S., Wang, Y., Ogasawara, K., Ogawa, A., 2016. Oxygen extraction fraction measurement using quantitative susceptibility mapping: comparison with positron emission tomography. J. Cereb. Blood Flow Metab. 36, 1424-1433. doi:10.1177/0271678X15606713.

Lakens, D., 2013. Calculating and reporting effect sizes to facilitate cumulative science: a practical primer for t-tests and ANOVAs. Front. Psychol. 4, 863. doi:10.3389/fpsyg.2013.00863.

Langkammer, C., Bredies, K., Poser, B.A., Barth, M., Reishofer, G., Fan, A.P., Bilgic, B., Fazekas, F., Mainero, C., Ropele, S., 2015. Fast quantitative susceptibility mapping using 3D EPI and total generalized variation. Neuroimage 111, 622-630. doi:10.1016/j.neuroimage.2015.02.041

Langkammer, C., Schweser, F., Shmueli, K., Kames, C., Li, X., Guo, L., Milovic, C., Kim, J., Wei, H., Bredies, K., 2018. Quantitative susceptibility mapping: Report from the 2016 reconstruction challenge. Magn. Reson. Med. 79, 1661-1673. doi: $10.1002 / \mathrm{mrm} .26830$.

Law, M.W., Chung, A.C., 2013. Segmentation of intracranial vessels and aneurysms in phase contrast magnetic resonance angiography using multirange filters and local variances. IEEE Trans. Image Process 22, 845-859. doi:10.1109/TIP.2012.2216274.
Li, W., Wang, N., Yu, F., Han, H., Cao, W., Romero, R., Tantiwongkosi, B., Duong, T.Q., Liu, C., 2015. A method for estimating and removing streaking artifacts in quantitative susceptibility mapping. Neuroimage 108, 111-122. doi:10.1016/j.neuroimage.2014.12.043.

Li, W., Wu, B., Liu, C., 2011. Quantitative susceptibility mapping of human brain reflects spatial variation in tissue composition. Neuroimage 55, 1645-1656. doi:10.1016/j.neuroimage.2010.11.088.

Liu, J., Liu, T., de Rochefort, L., Ledoux, J., Khalidov, I., Chen, W., Tsiouris, A.J., Wisnieff, C., Spincemaille, P., Prince, M.R., Wang, Y., 2012. Morphology enabled dipole inversion for quantitative susceptibility mapping using structural consistency between the magnitude image and the susceptibility map. Neuroimage 59, 2560-2568. doi:10.1016/j.neuroimage.2011.08.082.

Liu, T., Khalidov, I., de Rochefort, L., Spincemaille, P., Liu, J., Tsiouris, A.J., Wang, Y., 2011. A novel background field removal method for MRI using projection onto dipole fields (PDF). NMR Biomed. 24, 1129-1136. doi:10.1002/nbm.1670.

Liu, T., Wisnieff, C., Lou, M., Chen, W., Spincemaille, P., Wang, Y., 2013. Nonlinear formulation of the magnetic field to source relationship for robust quantitative susceptibility mapping. Magn. Reson. Med. 69, 467-476. doi:10.1002/mrm.24272.

Liu, Z., Kee, Y., Zhou, D., Wang, Y., Spincemaille, P., 2017. Preconditioned total field inversion (TFI) method for quantitative susceptibility mapping. Magn. Reson. Med. 78, 303-315. doi:10.1002/mrm.26331.

Marques, J.P., Bowtell, R., 2005. Application of a Fourier-based method for rapid calculation of field inhomogeneity due to spatial variation of magnetic susceptibility. In: Concepts in Magnetic Resonance Part B: Magnetic Resonance Engineering 25B, pp. 65-78. doi:10.1002/cmr.b.20034.

McFadden, J.J., Matthews, J.C., Scott, L.A., Parker, G.J., Lohézic, M., Parkes, L.M., 2021. Optimization of quantitative susceptibility mapping for regional estimation of oxygen extraction fraction in the brain. Magn. Reson. Med. doi:10.1002/mrm.28789.

Mintun, M.A., Raichle, M.E., Martin, W.R., Herscovitch, P., 1984. Brain oxygen utilization measured with O-15 radiotracers and positron emission tomography. J. Nucl. Med. 25, 177-187.

Pruessmann, K.P., Weiger, M., Scheidegger, M.B., Boesiger, P., 1999. SENSE: sensitivity encoding for fast MRI. Magn. Reson. Med. 42, 952-962 https://doi.org/10.1002/(sici)1522-2594(199911)42:5<952::aid-mrm16>3.0.co;2-s.

Robinson, S.D., Bredies, K., Khabipova, D., Dymerska, B., Marques, J.P., Schweser, F., 2017. An illustrated comparison of processing methods for MR phase imaging and QSM: combining array coil signals and phase unwrapping. NMR Biomed. 30, e3601. doi:10.1002/nbm.3601.

Schneider, T.M., Möhlenbruch, M., Denoix, M., Ladd, M.E., Bendszus, M., Heiland, S., Straub, S., 2020. Susceptibility-based characterization of cerebral arteriovenous malformations. Invest. Radiol. 55, 702-710. doi:10.1097/RLI.0000000000000695.

Schofield, M.A., Zhu, Y., 2003. Fast phase unwrapping algorithm for interferometric applications. Opt. Lett. 28, 1194-1196. doi:10.1364/ol.28.001194.

Schweser, F., Deistung, A., Sommer, K., Reichenbach, J.R., 2013. Toward online reconstruction of quantitative susceptibility maps: superfast dipole inversion. Magn. Reson. Med. 69, 1582-1594. doi:10.1002/mrm.24405.

Schweser, F., Robinson, S.D., de Rochefort, L., Li, W., Bredies, K., 2017. An illustrated comparison of processing methods for phase MRI and QSM: removal of background field contributions from sources outside the region of interest. NMR Biomed. 30, e3604. doi:10.1002/nbm.3604.

Spees, W.M., Yablonskiy, D.A., Oswood, M.C., Ackerman, J.J., 2001. Water proton MR properties of human blood at 1.5 Tesla: Magnetic susceptibility, T1, T2, T, and nonLorentzian signal behavior. Magn. Reson. Med. 45, 533-542. doi:10.1002/mrm.1072.

Stolz, E., Kaps, M., Kern, A., Babacan, S.S., Dorndorf, W., 1999. Transcranial color-coded duplex sonography of intracranial veins and sinuses in adults: reference data from 130 volunteers. Stroke 30, 1070-1075. doi:10.1161/01.STR.30.5.1070.

Straub, S., Schneider, T.M., Emmerich, J., Freitag, M.T., Ziener, C.H., Schlemmer, H.P., Ladd, M.E., Laun, F.B., 2017. Suitable reference tissues for quantitative susceptibility mapping of the brain. Magn. Reson. Med. 78, 204-214. doi:10.1002/mrm.26369.

Ward, P.G., Fan, A.P., Raniga, P., Barnes, D.G., Dowe, D.L., Ng, A.C., Egan, G.F., 2017. Improved quantification of cerebral vein oxygenation using partial volume correction. Front. Neurosci. 11, 89. doi:10.3389/fnins.2017.00089.

Wei, H., Dibb, R., Zhou, Y., Sun, Y., Xu, J., Wang, N., Liu, C., 2015. Streaking artifact reduction for quantitative susceptibility mapping of sources with large dynamic range. NMR Biomed. 28, 1294-1303. doi:10.1002/nbm.3383.

Weisskoff, R.M., Kiihne, S., 1992. MRI susceptometry: image-based measurement of absolute susceptibility of MR contrast agents and human blood. Magn. Reson. Med. 24, 375-383. doi:10.1002/mrm.1910240219.

Wu, B., Li, W., Avram, A.V., Gho, S.-M., Liu, C., 2012a. Fast and tissue-optimized mapping of magnetic susceptibility and T2* with multi-echo and multi-shot spirals. Neuroimage 59, 297-305. doi:10.1016/j.neuroimage.2011.07.019.

Wu, B., Li, W., Guidon, A., Liu, C., 2012b. Whole brain susceptibility mapping using compressed sensing. Magn. Reson. Med. 67, 137-147. doi:10.1002/mrm.23000.

Xu, B., Liu, T., Spincemaille, P., Prince, M., Wang, Y., 2014. Flow compensated quantitative susceptibility mapping for venous oxygenation imaging. Magn. Reson. Med. 72, 438-445. doi:10.1002/mrm.24937.

Yushkevich, P.A., Piven, J., Hazlett, H.C., Smith, R.G., Ho, S., Gee, J.C., Gerig, G., 2006. User-guided 3D active contour segmentation of anatomical structures: significantly improved efficiency and reliability. Neuroimage 31, 1116-1128. doi:10.1016/j.neuroimage.2006.01.015. 